\title{
ARTICLE Modulation of intestinal microbiota by glycyrrhizic acid prevents high-fat diet-enhanced pre-metastatic niche formation and metastasis
}

\author{
Miao Qiu ${ }^{1}$, Keqing Huang ${ }^{1}$, Yanzhuo Liu ${ }^{1}$, Yuqing Yang ${ }^{2}$, Honglin Tang ${ }^{1}$, Xiaoxiao Liu ${ }^{1}$, Chenlong Wang ${ }^{1}$, Honglei Chen $^{3}$, Yu Xiong ${ }^{4}$, \\ Jing Zhang ${ }^{5}$ and Jing Yang ${ }^{1}$
}

High-fat diet (HFD) promotes lung pre-metastatic niche formation and metastasis. Thus, there is an urgent need to identify the underlying mechanisms and develop strategies to overcome them. Here we demonstrate that glycyrrhizic acid (GA) prevents HFDenhanced pre-metastatic niche formation and metastasis through gut microbiota. GA reduced HFD-enhanced myeloid-derived suppressor cell recruitment, pro-metastatic protein S100A8/A9 expression and metastasis burden of 4T1 breast cancer and B16F10 melanoma, accompanied by gut microbiota alteration and colonic macrophage polarization far away the M1-like phenotype. These parameters were greatly decreased by treatment with antibiotics, recolonization of Desulfovibrio vulgaris and Clostridium sordellii, and administration of lipopolysaccharide or deoxycholic acid. Macrophage depletion attenuated HFD-enhanced pre-metastatic niche formation and metastasis, but failed to further affect the effects of GA. Mechanistically, counteraction of HFD-enhanced gut microbiota dysbiosis by GA inhibited $\mathrm{Gr}-1^{+}$myeloid cell migration and S100A8/A9 expression through decreasing the proportion of M1-like macrophages and their production of CCL2 and TNF- $\alpha$ in the colons via LPS/HMGB1/NF-KB signaling inactivation. Together, targeting the gut microbiota by GA to modulate colonic macrophages could be a novel strategy for the prevention of HFDenhanced pre-metastatic niche formation and metastasis.

Mucosal Immunology (2019) 12:945-957; https://doi.org/10.1038/s41385-019-0144-6

\section{INTRODUCTION}

Tumor metastasis is responsible for $90 \%$ of cancer-related mortality. ${ }^{1}$ The tumor-derived factors including tumor necrosis factor-a (TNF-a) and CC chemokine ligand 2 (CCL2) induce the supportive metastatic environments in the secondary sites before arrival of tumor cells, termed as the pre-metastatic niche. ${ }^{2}$ The recruitment of bone marrow-derived cells such as myeloid-derived suppressor cells (MDSCs) and CD11 ${ }^{+}$myeloid cells initiates the pre-metastatic niches and thereby greatly increases the possibility of metastasis. ${ }^{3}$ Targeting the pre-metastatic niche-promoting molecules or cellar components may be an attractive approach for prevention of metastasis. ${ }^{4}$

Obesity is a risk factor for breast cancers, advanced malignant melanoma and colorectal carcinoma. ${ }^{5}$ Diet-induced obesity is increased dramatically over the past three decades in both developed and developing countries, and globally the fifth major cause of mortality. ${ }^{6}$ Prolonged high-fat diet (HFD) feeding induced obesity, and associated with the increased incidence or mortality for breast cancer and melanoma in addition to gastrointestinal tumors in murine models. ${ }^{7,8}$ HFD increases growth and metastasis of 4T1 mammary carcinoma and melanoma. ${ }^{9,10}$ HFD-induced obesity in C57BL/6 mice promotes breast cancer metastasis through lung pre-metastatic niche formation. ${ }^{11}$ However, the role of HFD in pre-metastatic niche formation has only begun to be appreciated.

HFD-induced changes in the composition of gut bacterial community contribute to intestinal carcinogenesis. ${ }^{12}$ The fecal microbiota from the patients with colorectal cancer promotes tumorigenesis in germ-free mice and carcinogen-treated mice, accompanied with the upregulation of tumor invasiveness and metastasis related-genes. ${ }^{13}$ The gut microbiota promotes liver metastasis through translocation of bacterial to the metastatic site. ${ }^{14}$ Modulation of gut microbiota by probiotics suppresses hepatocellular carcinoma growth. ${ }^{15}$ Bacterial lipopolysaccharide (LPS)-induced inflammation increases melanoma lung metastasis, ${ }^{16}$ and gut microbial metabolite deoxycholic acid (DCA) promotes hepatocellular carcinoma development. ${ }^{17}$ Thus, downregulation of LPS and DCA from gut microbiota may be potential therapeutic strategies for HFD-enhanced metastasis.

The macrophage is one of the most abundant leukocytes in the intestines of mammals. ${ }^{18}$ The recruitment of macrophages into lung pre-metastatic niche contributes to HFD-enhanced breast cancer metastasis. ${ }^{19}$ Functionally differentiated macrophages are commonly subdivided into two categories, the classically activated

\footnotetext{
${ }^{1}$ Department of Pharmacology and Hubei Province Key Laboratory of Allergy and Immune-Related Diseases, School of Basic Medical Sciences, Wuhan University, 430071 Wuhan, China; ${ }^{2}$ Department of Pharmaceutics, Ernest Mario School of Pharmacy, Rutgers, The State University of New Jersey, Piscataway, NJ, USA; ${ }^{3}$ Department of Pathology and Pathophysiology, School of Basic Medical Sciences, Wuhan University, 430071 Wuhan, China; ${ }^{4}$ Department of Radiation and Medical Oncology, Zhongnan Hospital, Wuhan University, 430071 Wuhan, China and ${ }^{5}$ Animal Experimental Center of Wuhan University, 430071 Wuhan, China

Correspondence: Jing Yang (jing_yang@whu.edu.cn)

These authors contributed equally: Miao Qiu, Keqing Huang
}

Received: 26 August 2018 Revised: 9 January 2019 Accepted: 27 January 2019

Published online: 12 February 2019 
946

M1-like phenotype, which produces pro-inflammatory factors and anti-tumor response, and the alternatively activated M2-like phenotype, which produces anti-inflammatory factors and protumor process. $^{20}$ Recent studies show that the M1-like macrophages enhance metastatic potential of colon, hepatocellular, and ovarian cancer cells. ${ }^{21-23}$ The high-mobility group box (HMGB1) contributes to melanoma growth and lung metastasis formation, and the release of HMGB1 induced by LPS facilitates M1-like phenotype through the nuclear factor kappa B (NF-KB) signaling. ${ }^{24,25}$ Gut dysbiosis induced by a maternal western-style diet (high fat and high sugar) polarizes bone marrow-derived macrophages to an inflammatory state. ${ }^{26}$ However, whether modulation of the macrophage response by reshaping gut microbiota prevents pre-metastatic niche formation and metastasis is not fully understood.

Saponins, a novel type of plant-derived secondary metabolites, modulate gut microbiota composition and exhibit anti-metastasis activities in multiple tumors including lung adenocarcinoma, hepatocellular carcinoma, and leukemia. ${ }^{27,28}$ Glycyrrhizic acid (GA), a triterpenoid saponin glycoside from the licorice roots, inhibits tumor growth and B16 melanoma pulmonary metastasis. ${ }^{29,30} \mathrm{GA}$ also ameliorates HFD-induced obesity and non-alcoholic fatty liver disease in murine models. ${ }^{31,32} \mathrm{GA}$ exhibits the anti-inflammatory properties and tissue protective properties by binding to HMGB1 and inhibiting its cytokine activities. ${ }^{33}$ However, to date, whether GA prevents HFD-mediated gut dysbiosis and pre-metastatic niche formation has not been reported. Here, we demonstrated that modulation of gut microbiota by GA prevents HFD-enhanced pre-metastatic niche formation and metastasis through decreasing M1-like colonic macrophages via LPS/HMGB1/NF-KB signaling. Our results may provide a potential therapeutic strategy for prevention of metastasis.

\section{RESULTS}

GA inhibits HFD-enhanced lung pre-metastatic niche formation, and thus prevents metastasis

We first investigated whether GA prevents HFD-mediated metastasis on day 28 after implantation of tumor cells to the HFD-fed mice. As shown in Fig. $1 a$ and $b$, the lung luciferase intensity and metastatic nodules of $4 \mathrm{~T} 1$ breast cancer or B16F10 melanoma cells were significantly increased in the HFD-fed mice as compared with the control. Conversely, GA (10 and $20 \mathrm{mg} / \mathrm{kg}$ ) decreased lung luciferase intensity and metastatic nodules in the HFD-fed mice. Next, we measured the effects of GA on the expression of pro-metastatic proteins and the infiltration of MDSCs in the pre-metastatic lungs from the HFD-treated mice. As shown in Fig. 1c, pro-metastatic proteins matrix metalloproteinase-9 (MMP-9) and S100A8/A9 in the lungs from the HFD-treated mice were significantly upregulated as compared with the control diet mice. Conversely, GA (10 and $20 \mathrm{mg} / \mathrm{kg}$ ) significantly inhibited MMP-9 and S100A8/A9 expression in the lungs. Furthermore, HFD significantly elevated the percentage of $\mathrm{CD} 11 \mathrm{~b}^{+} \mathrm{Ly}_{6 \mathrm{G}}{ }^{+}$granulocytic MDSCs (G-MDSCs) and CD11 $\mathrm{b}^{+}$Ly6C $\mathrm{C}^{+}$monocytic MDSCs (MMDSCs) in the lungs, which were inhibited by GA (Fig. 1d, e). We found that the arginase activity of the $\mathrm{Gr}-1^{+} \mathrm{MDSCs}$ in the lung was significantly increased by the HFD, but decreased by the GA (Fig. 1f). The percent of $\mathrm{CD} 8^{+} \mathrm{T}$ cells in the lungs was lower in the HFD-fed mice than in the control mice, whereas, the percent of $\mathrm{CD}^{+} \mathrm{T}$ cells was higher in the GA-treated mice than in the HFDfed mice (Fig. 1g). We performed co-culture experiments of the $\mathrm{Gr}$ $1^{+}$MDSCs from the lungs of HFD and GA-fed mice with the CD8 ${ }^{+}$ $T$ cells, and found that Gzm B, IFN- $\gamma$, and IL-2 production in the $\mathrm{CD}^{+} \mathrm{T}$ cells was decreased by the $\mathrm{Gr}-1^{+}$MDSCs from the HFD-fed mice, but increased by the Gr-1 $1^{+}$MDSCs from the GA-fed mice (Fig. 1h). We monitored the body weights and food intake of all mice throughout the study, and found that the HFD increased body weight and food intake of mice, which were not affected by the GA (Fig. 1i, j). Similar results were obtained in the C57BL/6 mice (Fig. S1).

Modulation of gut microbiota by GA prevents HFD-induced premetastatic niche formation and metastasis

To investigate whether GA modulates HFD-mediated gut microbiota dysbiosis, we sequenced the $16 \mathrm{~S}$ bacterial gene. UniFracbased principal coordinates analysis revealed obvious difference in a distinct clustering of fecal microbial structure among the control, HFD and GA groups (Fig. 2a). This was further supported by the obvious different abundance of bacterial phyla among the three groups (Fig. 2b). The linear discriminant analysis effect size (LEfSe) showed that Clostridiales order and Desulfovibrio genus predominantly acted to HFD-mediated gut microbiota dysbiosis (Fig. 2c, d). Conversely, GA significantly decreased Clostridiales order and Desulfovibrio genus in the HFD-treated mice (Fig. S3A). LPS and DCA levels in the feces and peripheral blood were significantly increased by the HFD but decreased by the GA (Fig. $2 e$ and S2A-B). The ratio between Firmicutes and Bacteroidetes, a marker of gut dysbiosis, was also increased by the HFD but reduced by the GA (Fig. 2f). To clarify whether gut microbiota is crucial for the effects of GA on HFD-induced pre-metastatic niche formation and metastasis, we used a cocktail of broadspectrum antibiotics (Abx) to deplete gut microbiota. qPCR analysis of total bacterial 16S rRNA genes confirmed the antibacterial efficacy of Abx (Fig. 3a, b). As we expected, Abx treatment attenuated the effects of HFD and GA on the levels of LPS and DCA in the feces and peripheral blood, the infiltration of MDSCs and the number of metastatic nodules in the lungs (Fig. 3c-f). These finding indicates that GA decreases LPS and DCA levels in the feces and peripheral blood through the gut microbiota.

Desulfovibrio vulgaris (Des) is the predominant specie of Desulfovibrio genus in the human and mice colon, ${ }^{34,35}$ and Clostridium sordellii (Clo) composes the main DCA-producing strain in the HFD mice. ${ }^{17}$ We found that Des plus Clo were significantly increased by the HFD but decreased by the GA (Fig. S3A). To demonstrate directly that Des plus Clo are the main driver of premetastatic niche formation and metastasis, we colonized the Des plus Clo into the Balb/c mice (Fig. $3 \mathrm{~g}$ ). Colonization of Des plus Clo was confirmed by qPCR (Fig. S3B). As shown in Fig. 3h-l, the colonization of Des plus Clo elevated the levels of LPS and DCA in the feces and peripheral blood, promoted the infiltration of MDSCs and increased the number of metastatic nodules in the lungs.

GA regulates HFD-mediated alteration in colonic macrophage phenotype

We found that HFD induced much higher gene expression levels of iNOS and CXCL9 (M1 markers) and slightly higher gene expression levels of arginase-1 and CD206 (M2 markers) in the macrophage of colon (Fig. 4a, b). Conversely, GA significantly decreased iNOS and CXCL9 levels, and tended to inhibit the mRNA expression of arginase- 1 and CD206 though the difference did not reach significance (Fig. $4 \mathrm{a}, \mathrm{b}$ ). $\mathrm{CD}^{+} \mathrm{8}^{+}$is commonly used as a macrophage marker in the colon. ${ }^{36,37}$ HFD elevated $\mathrm{CD}^{+}{ }^{+}$iNOS $^{+}$ (M1-like) macrophages (about 3.5-fold) and $\mathrm{CD}^{+} 8^{+} \mathrm{CD} 206^{+}$(M2like) macrophages (about 2.0-fold) in the colons. Conversely, GA $(20 \mathrm{mg} / \mathrm{kg}$ ) significantly decreased M1-like macrophages, and tended to inhibit M2-like macrophage in the colons though the difference did not reach significance (Fig. 4c-e). Next, we measured the cytokines in the macrophage of colon, and found that the production of M1 cytokines (TNF- $a$ and CCL2) and M2 cytokine (TGF- $\beta$ ) were significantly elevated by the HFD but attenuated by the GA, while HFD and GA did not affect IL-10 (M2 cytokine) level (Fig. 4f, g). The release of HMGB1 induced by LPS facilitates M1-like phenotype. ${ }^{25}$ We found that the HMGB1 level in the peripheral blood was significantly increased by the HFD but decreased by the GA (Fig. 4h). 
a

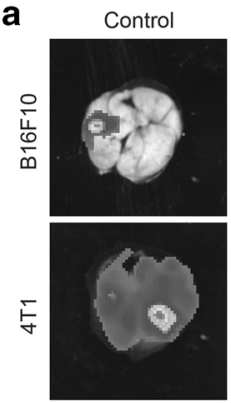

HFD
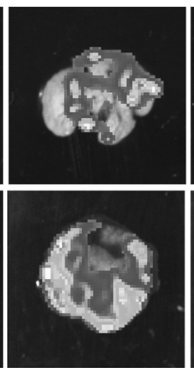

$\mathrm{GA}(10 \mathrm{mg} / \mathrm{kg})$
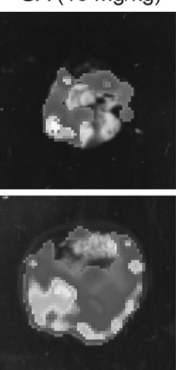

$\mathrm{GA}(20 \mathrm{mg} / \mathrm{kg})$

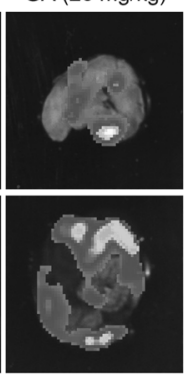

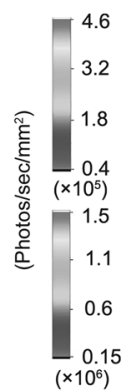

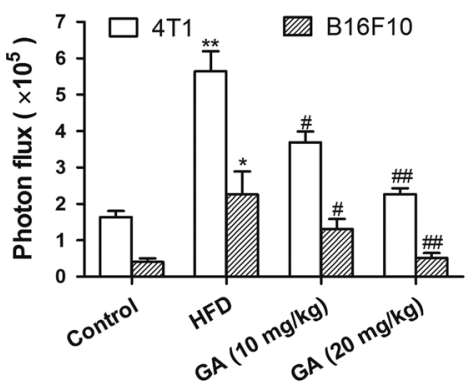

b

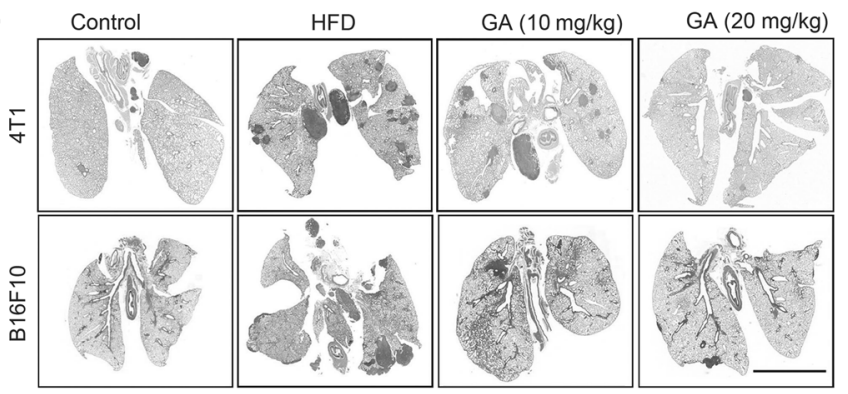

C

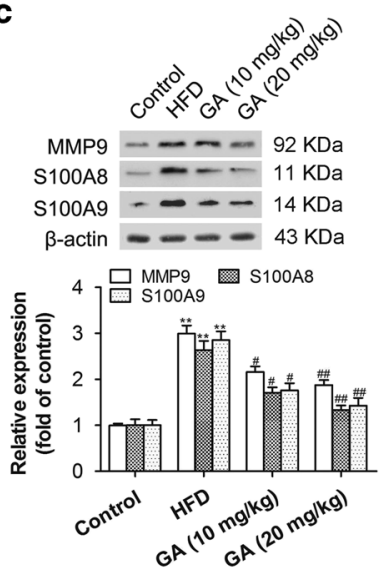

d
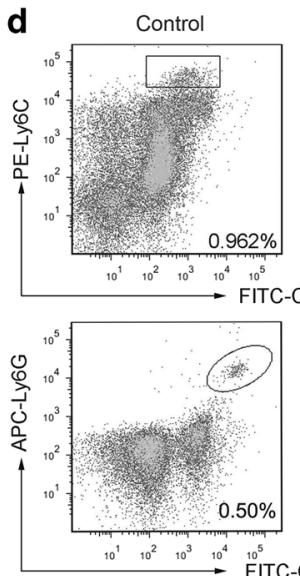
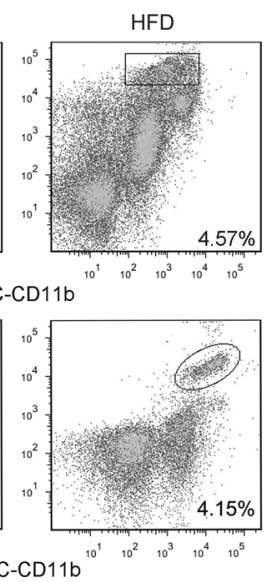
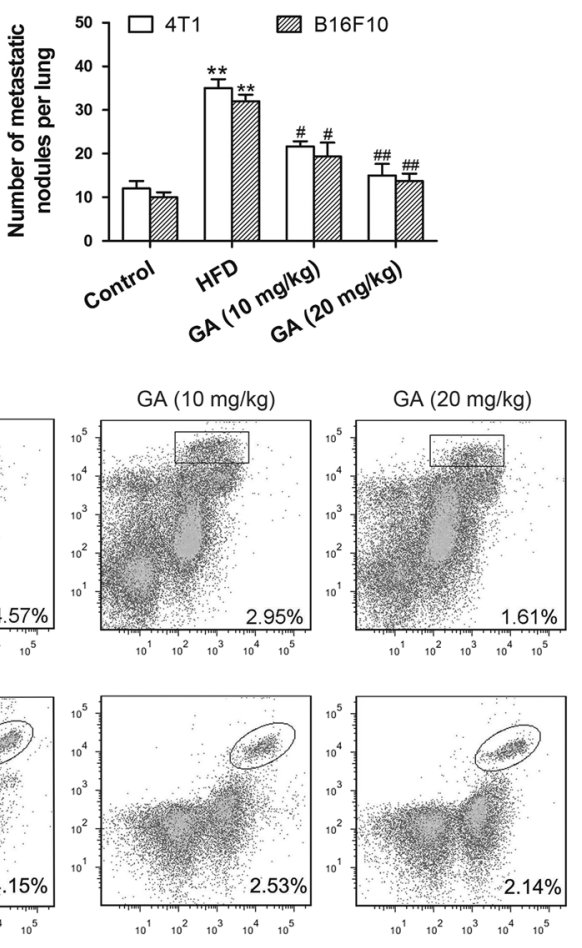

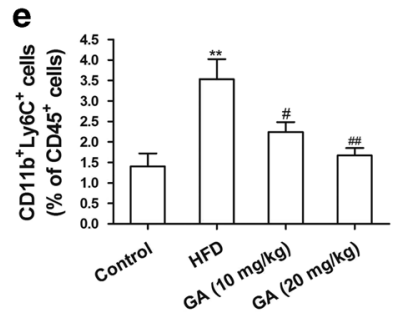

h

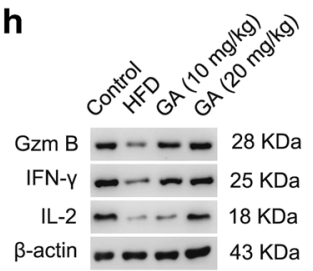

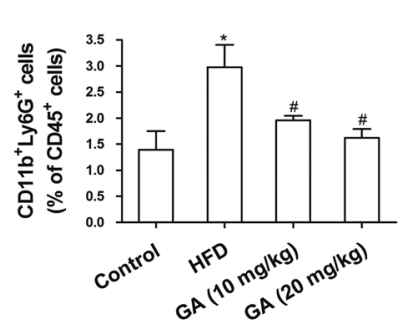
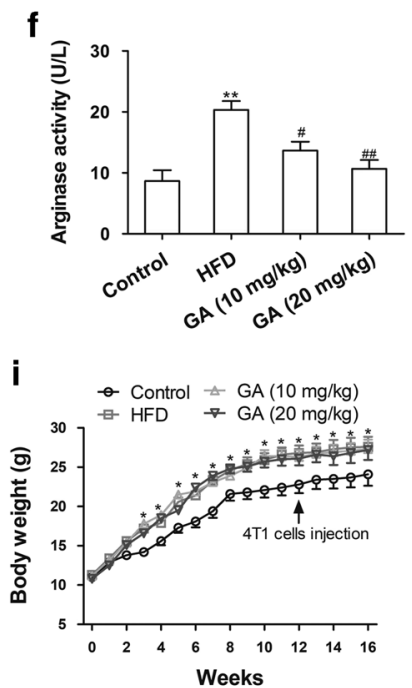
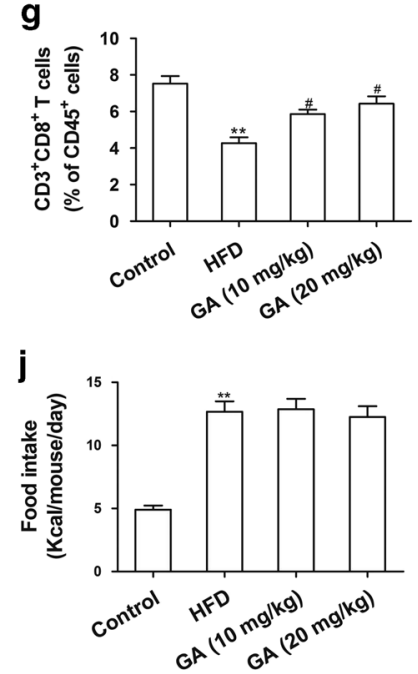

GA-modulated gut microbiota alters macrophage phenotypes and their production of cytokines

Next, we transferred the commercially available cocktail of Des plus Clo, or supplemented $0.2 \%$ DCA or LPS $(300 \mu \mathrm{g} / \mathrm{kg})$ into the GA-treated mice (Fig. 5a). Colonization of Des plus Clo was confirmed by qPCR (Fig. S4A). Des plus Clo increased LPS and DCA levels in the feces and peripheral blood (Fig. 5b and S4B). Administration of Des plus Clo, LPS and DCA induced HMGB1 translocation from nucleus to cytoplasm (Fig. 5c, d). Des plus Clo induced the expression of M1 markers (iNOS and CXCL9) without 
Fig. 1 Glycyrrhizic acid (GA) inhibits high-fat diet (HFD)-enhanced lung pre-metastatic niche formations, and thus prevents metastasis. The Balb/c mice or C57BL/6 mice were treated with the control diet, HFD and HFD plus GA ( 10 and $20 \mathrm{mg} / \mathrm{kg}$, i.g., qd $\times 5$ days per w) for $12 \mathrm{w}$. 4 T1luciferase cells $\left(1 \times 10^{6}\right)$ or B16F10-luciferase cells $\left(5 \times 10^{5}\right)$ were then injected to the right mammary fat pad of Balb/c mice and the armpit of C57BL/6 mice, respectively. a At $4 \mathrm{w}$ after tumor implantation, representative images and quantitative analysis of lung metastasis were detected by ex vivo luciferase-based bioluminescence imaging $(n=8)$. $\mathbf{b}$ Hematoxylin/eosin-stained lung sections and quantification of lung metastases $(n=8)$. Scale bars, $5 \mathrm{~mm}$. c The mice were treated with the control diet, HFD and HFD plus GA for $12 \mathrm{w}$, MMP-9, S100A8, and S100A9 in the lungs from the Balb/c mice were determined by western blot $(n=8)$. d, e CD11 b ${ }^{+}{\text {Ly } 6 G^{+}}$and CD $11 b^{+}$Ly6C $^{+}$myeloid cells in the lungs from the Balb/c mice were determined by flow cytometry $(n=5)$. $f$ The arginase activity in the $\mathrm{Gr}-1^{+}$cells from the lung tissues was determined $(n=8)$. $\mathbf{g}$ The percent of $\mathrm{CD}^{+}$T cells in the pre-metastatic lungs was determined by flow cytometry $(n=5)$. $\mathbf{h}$ Gzm B, IFN- $\gamma$ and IL2 in $\mathrm{CD}^{+}$T cells were determined by western blot. $\mathbf{i}, \mathbf{j}$ Changes in body weights and food intake $(n=8)$. The values are presented as the mean \pm SEM. ${ }^{*} P<0.05,{ }^{* *} P<0.01$ vs. control, ${ }^{\#} P<0.05,{ }^{\# \#} P<0.01$ vs. HFD
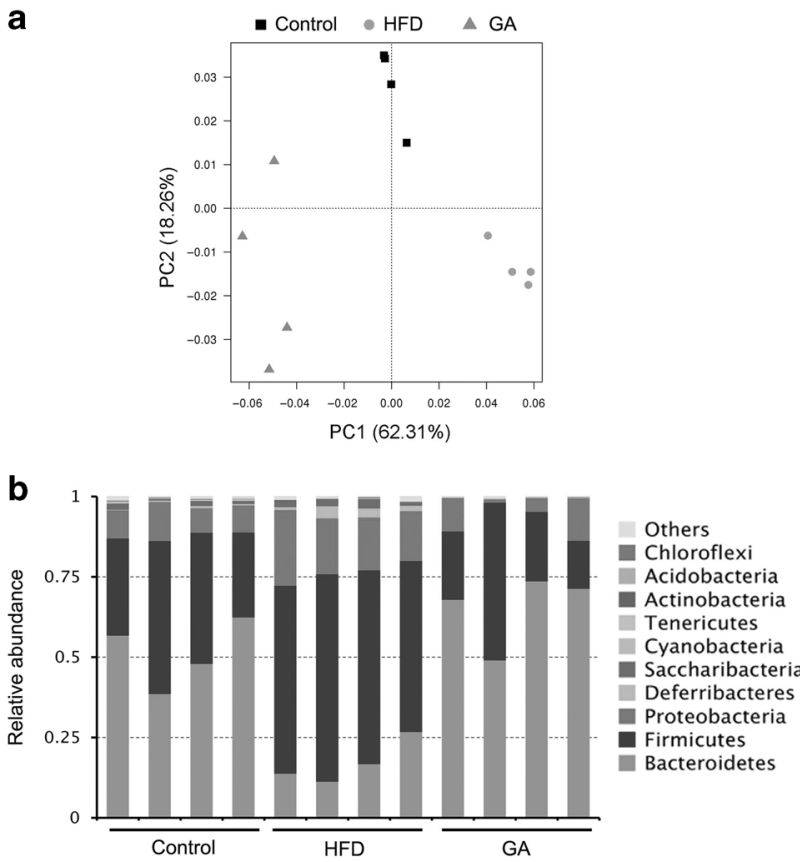

d
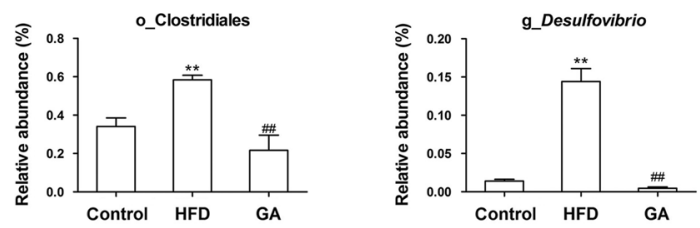

e
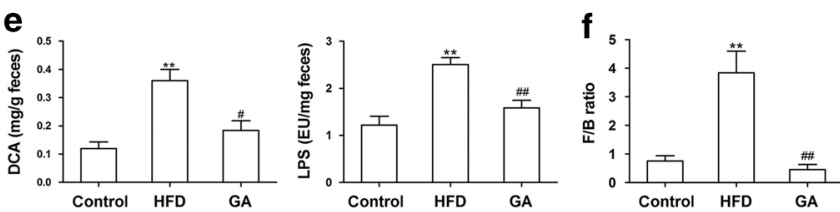

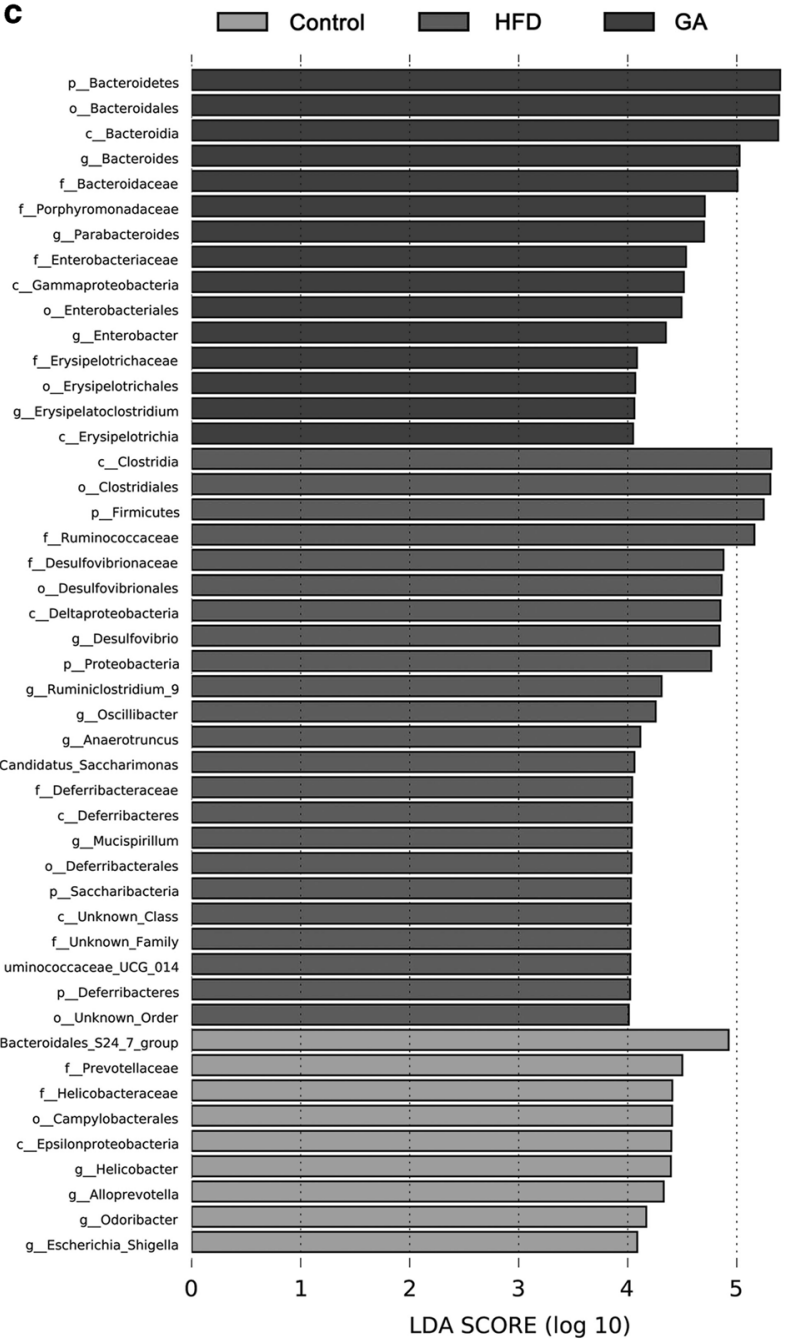

Fig. 2 Glycyrrhizic acid (GA) modulates high-fat diet (HFD)-induced alternation of microbial community. The Balb/c mice were treated with the control diet, HFD and HFD plus GA $(20 \mathrm{mg} / \mathrm{kg}$, i.g., $\mathrm{qd} \times 5$ days per w) for $12 \mathrm{w}$. a Principal coordinate analysis plot of bacterial $\beta$-diversity over time $(n=4)$. b The relative taxonomic abundance at the phylum level of gut microbiota. $(\%)$ in the fecal bacterial community $(n=4)$. c Linear discriminant analysis (LDA) scores derived from linear discriminant analysis effect size (LEfSe) analysis, showing the biomarker taxa, LDA threshold value of $>4.0$ were shown $(n=4)$. d Relative abundance of Clostridiales order and Desulfovibrio genus. e The levels of lipopolysaccharide (LPS) or deoxycholic acid (DCA) in the feces were determined by LC-MS or Limulus Amebocyte Lysate assays, respectively $(n=8)$. $f$ The ratios of Firmicutes/Bacteroidetes $(n=4)$. The values are presented as the mean \pm SEM. ${ }^{*} P<0.05,{ }^{* *} P<0.01$ vs. control, ${ }^{\sharp} P<0.05$, ${ }^{\#} P<0.01$ vs. HFD

significantly influencing the expression of M2 markers (arginase-1 and CD206) (Fig. 5e). Furthermore, LPS increased iNOS and CXCL9 levels and DCA increased iNOS and arginase-1, CD206 levels (Fig. 5e). We observed the similar alteration in the proportion of $\mathrm{CD}^{+}{ }^{+}$iNOS $^{+}$(M1-like) and $\mathrm{CD}^{+} 8^{+} \mathrm{CD}_{206}{ }^{+}$(M2-like) colonic macrophages by immunofluorescence (Fig. $5 f$ ). The supplementation of Des plus Clo, DCA or LPS increased the protein levels of pro-metastatic proteins (MMP-9 and S100A8/A9), the percentage of MDSCs (G-MDSCs and M-MDSCs) and the number of lung metastatic nodules (Fig. $5 \mathrm{~g}, \mathrm{~h}$ ).

Next, we determined the in vitro effects of the fecal extracts on the macrophage phenotype. As shown in Fig. $6 a$ and b, the fecal extracts from the HFD-fed mice significantly increased production of M1 cytokines (TNF- $\alpha$ and CCL2) and M2 cytokines (TGF- $\beta$ and IL- 


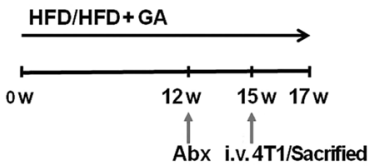

d

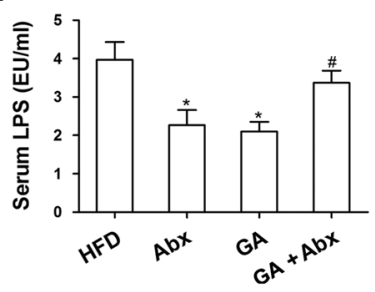

g

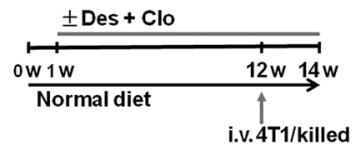

j

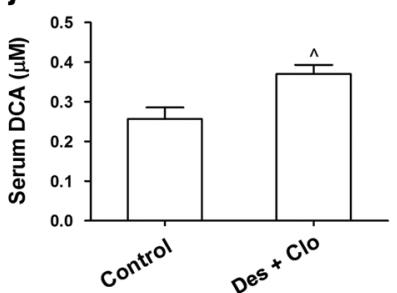

b
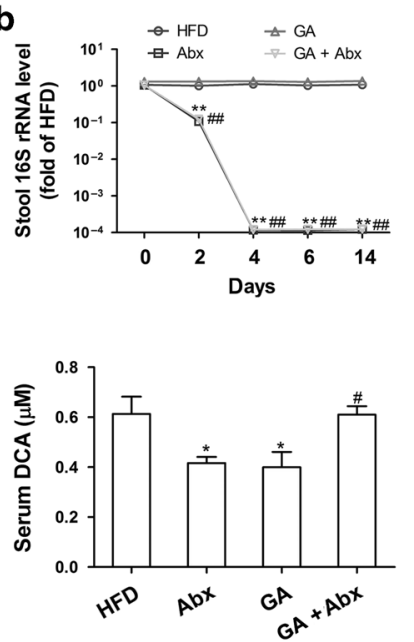

h

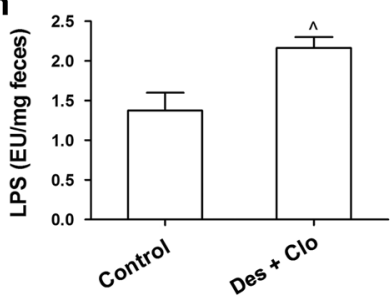

k

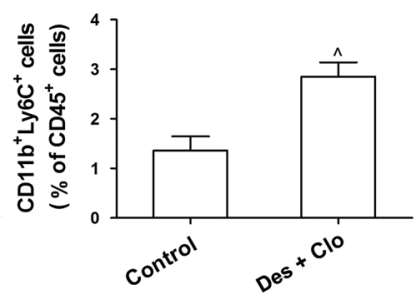

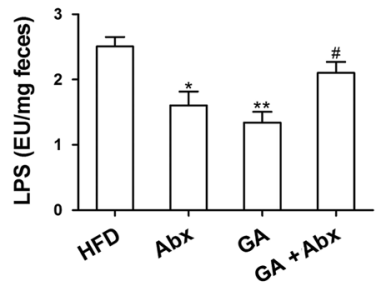
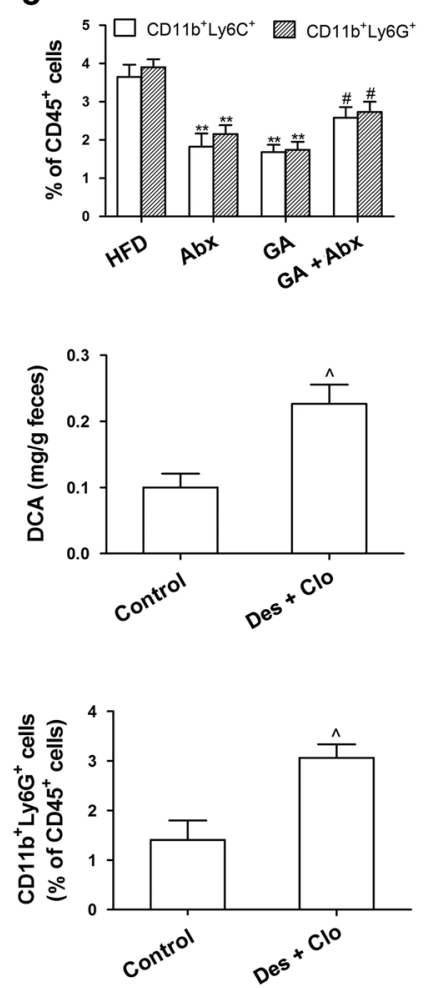
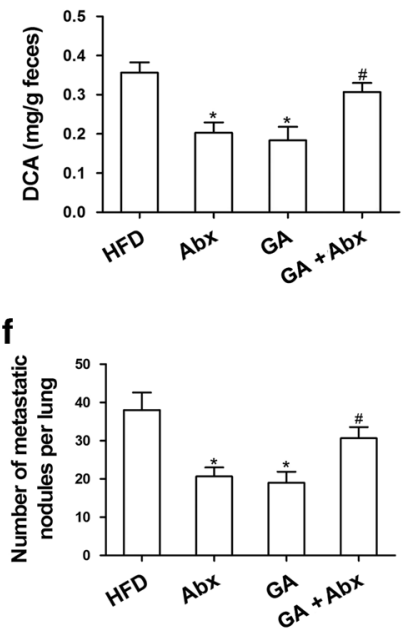

i

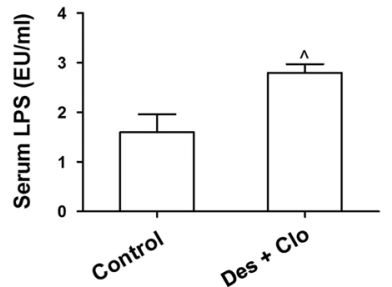

I

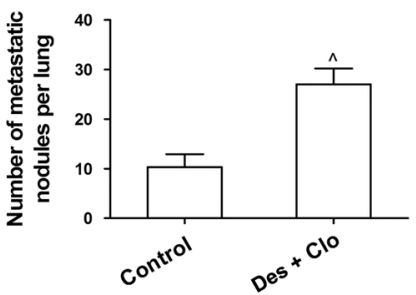

Fig. 3 Glycyrrhizic acid (GA) prevents high-fat diet (HFD)-enhanced lung pre-metastatic niche formation and metastasis through modulation of gut microbiota. a The Balb/c mice were received the HFD plus GA $(20 \mathrm{mg} / \mathrm{kg})$ or vehicle for $12 \mathrm{w}$, and were then treated with a broadspectrum antibiotic cocktail (Abx) consisting of $0.5 \mathrm{~g} / \mathrm{l}$ vancomycin, $0.5 \mathrm{~g} / \mathrm{l}$ neomycin sulfate, and $0.5 \mathrm{~g} / \mathrm{l}$ primaxin for $3 \mathrm{w}(n=8)$. $\mathbf{b}$ The efficacy of $\mathrm{Abx}$ to remove gut bacteria was confirmed by quantifying stool bacterial load $(n=8)$. c, d The levels of deoxycholic acid (DCA) or lipopolysaccharide (LPS) in the feces and peripheral blood were determined by LC-MS or Limulus Amebocyte Lysate assay, respectively ( $n=$ 8). e Myeloid-derived suppressor cells in the lungs were analyzed by flow cytometry $(n=5)$. f The mice were received HFD plus GA ( 20 mg/kg) or vehicle for $12 \mathrm{w}$, and then treated with Abx treatment for $3 \mathrm{w}$. 4T1 breast cancer cells $\left(2 \times 10^{5}\right)$ were injected via vein. At $2 \mathrm{w}$ after tumor injection, the number of lung metastatic nodules was counted. $\mathbf{g}$ The Balb/c mice were administered with bacterial suspension of Desulfovibrio vulgaris (Des) plus Clostridium sordellii (Clo) $\left(1 \times 10^{9} \mathrm{CFU} /\right.$ mouse, i.g., twice a week) or vehicle for $12 \mathrm{w}$. $\mathbf{h}-\mathbf{j}$ The levels of deoxycholic acid (DCA) or lipopolysaccharide (LPS) in the feces and peripheral blood were determined by LC-MS or Limulus Amebocyte Lysate assay, respectively $(n=8)$. k Myeloid-derived suppressor cells in the lungs were analyzed by flow cytometry $(n=5)$. I The mice were received Des plus Clo for 12 $\mathrm{W}$, and then injected $4 \mathrm{~T} 1$ breast cancer cells $\left(2 \times 10^{5}\right)$ via vein. At $2 \mathrm{w}$ after tumor injection, the number of lung metastatic nodules was counted. The values are presented as the mean \pm SEM. $\wedge P<0.05$ vs. control, ${ }^{*} P<0.05,{ }^{* *} P<0.01$ vs. HFD, ${ }^{\#} P<0.05$ vs. GA

10) in the RAW264.7 and THP-1 macrophages. Conversely, the fecal extracts from the GA-treated mice inhibited secretion of M1 cytokines without significantly influencing M2 cytokines. Consistently, the percent of M1-like macrophages was significantly increased by the fecal extracts from the HFD-fed mice, but attenuated by the fecal extracts from the GA-treated mice (Fig. 6c).

Modulation of colonic macrophage phenotype by GA prevents HFD-enhanced pre-metastatic niche formation and metastasis To clarify whether GA reprograms macrophage and thus prevents HFD-induced pre-metastatic niche formation and metastasis, we depleted macrophage by anti-CSF-1 antibody (IgG as control), and compared their effects on the HFD mice (containing more M1-like macrophages in the colons) with the GA-treated mice (containing less M1-like macrophages in the colons). Immunohistochemical analysis of $\mathrm{CD}^{+} 8^{+}$macrophages showed depletion of colonic macrophages by anti-CSF-1 antibody (Fig. 7a). Depletion of macrophages significantly decreased pre-metastatic niche formation (evidenced by a decrease in the MDSC recruitment and prometastatic protein expression) and the number of metastatic nodules in the lungs from the HFD-fed mice (Fig. $7 \mathrm{~b}-\mathrm{g}$ ), indicating that M1-like colonic macrophages promoted pre-metastatic niche formation and metastasis. However, macrophage depletion failed to further affect the inhibitory effects of GA on lung pre-metastatic niche formation and metastasis, indicating that GA regulated this process primarily through skewing colonic macrophages far from M1 phenotype. 
a

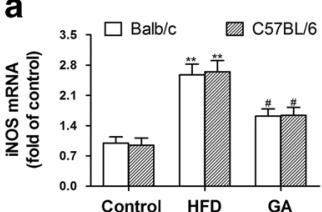

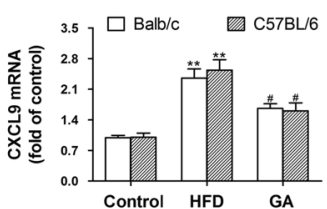

b
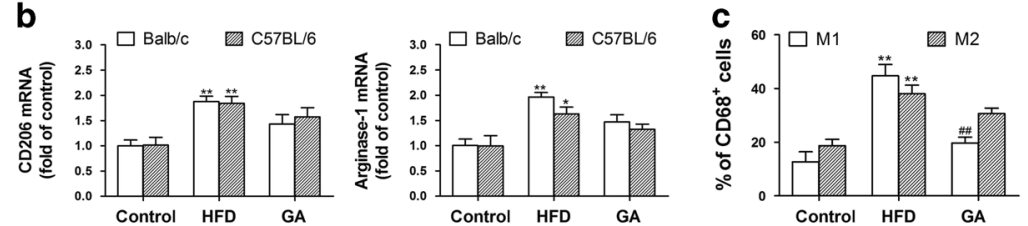

d
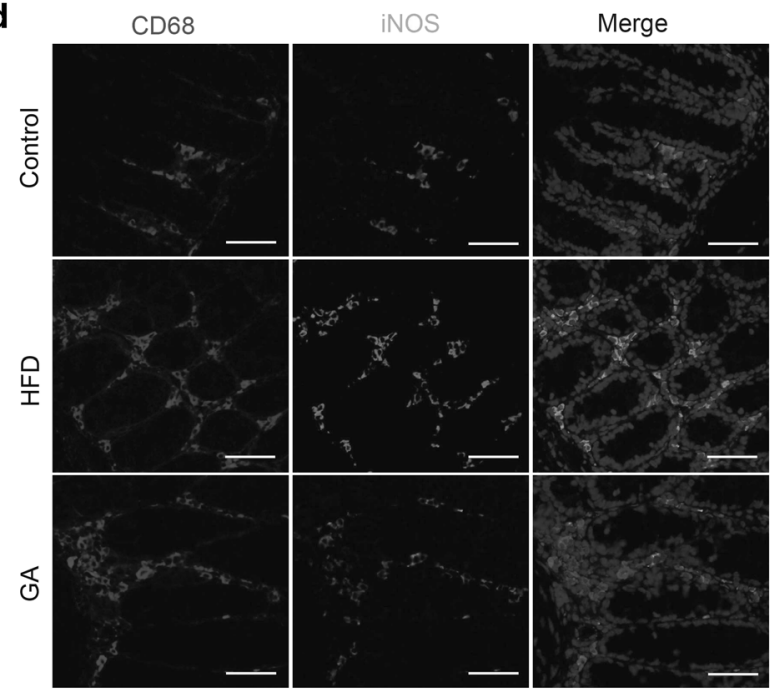

e
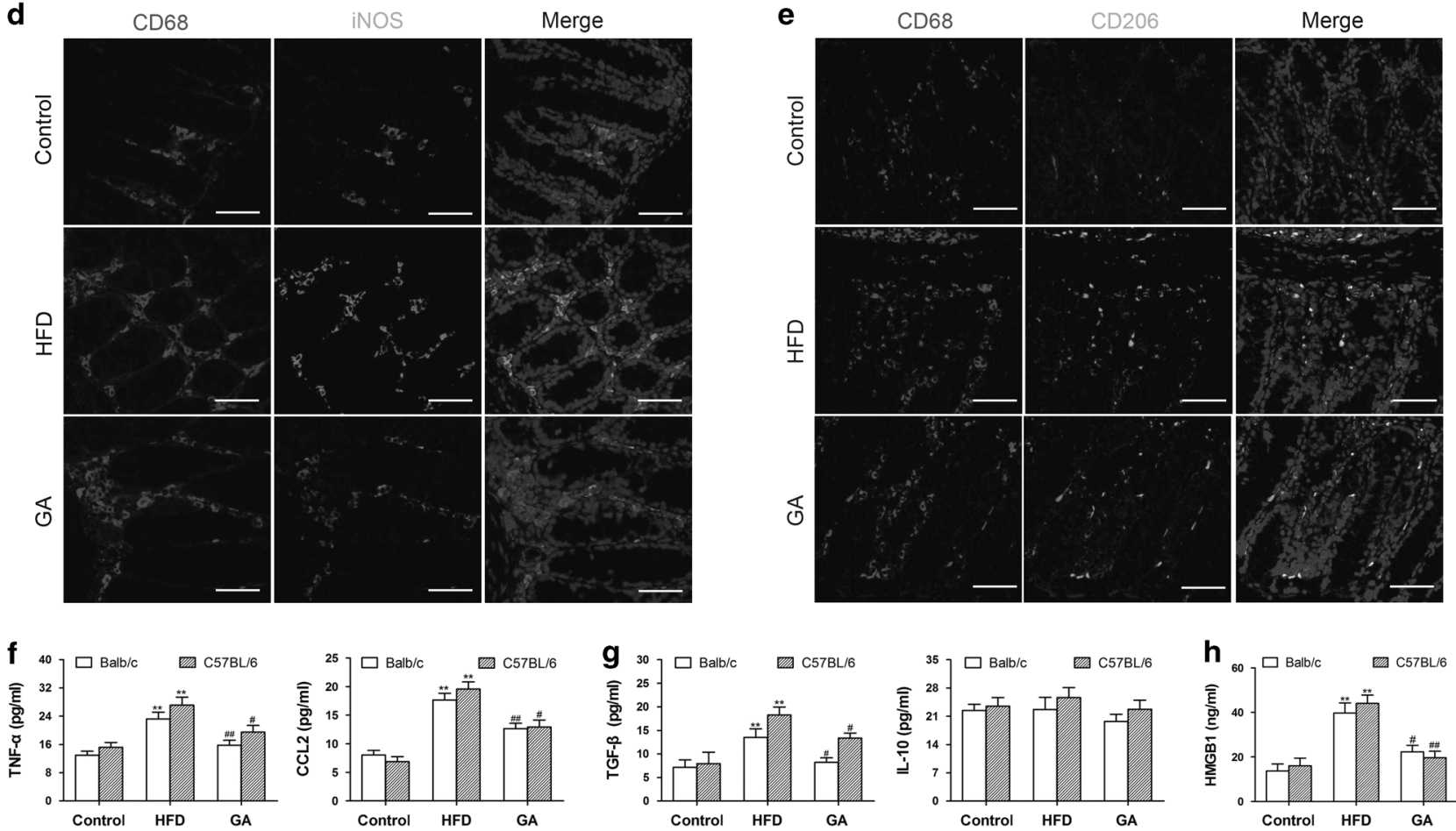

Fig. 4 Glycyrrhizic acid (GA) modulates high-fat diet (HFD)-induced colonic macrophage alternation and their cytokine production. The Balb/c mice or C57BL/6 mice treated with the control diet, HFD and HFD plus GA ( $20 \mathrm{mg} / \mathrm{kg}$, i.g., qd $\times 5$ days per w) for $12 \mathrm{w}$. a, b Relative mRNA levels of M1 markers (iNOS and CXCL9) and M2 markers (CD206 and arginase-1) in the colonic macrophages were measured by qPCR $(n=8)$. $c$ The ratio of M1- or M2-like colonic macrophages in the colon tissues from Balb/c mice $(n=8)$. d, e Immunostaining analysis of CD68 ${ }^{+}$iNOS $^{+}\left(\mathrm{M} 1-^{-}\right.$ like) and $\mathrm{CD}^{+} 8^{+} \mathrm{CD}^{206^{+}}$(M2-like) macrophages in the colon tissues from Balb/c mice was shown. Scale bars, $50 \mu \mathrm{m}$. $\mathbf{f}-\mathbf{g} \mathrm{M} 1 \mathrm{cytokines}$ (TNF- $\alpha$ and CCL2) and M2 cytokines (TGF- $\beta$ and IL-10) in the culture supernatants of colonic macrophages were determined by ELISA ( $n=8)$. $\mathbf{h}$ The level of high-mobility group box (HMGB1) in the peripheral blood was determined by ELISA. The values are presented as the mean \pm SEM. ${ }^{*} P<$ $0.05,{ }^{* *} P<0.01$ vs. control, ${ }^{\#} P<0.05,{ }^{\# \#} P<0.01$ vs. HFD

Colonic macrophage-derived factors regulated by GA inhibit premetastatic niche formation

Next, we determined whether colonic macrophage-derived cytokines could have a substantial impact on in vitro myeloid cell migration and S100A8/A9 expression, crucial steps for premetastatic niche formation. ${ }^{36}$ As shown in Fig. 8a, the conditioned media (CM) from colonic macrophages in the HFD-fed mice (HFD$\mathrm{CM})$ significantly increased the migration of $\mathrm{Gr}-1^{+}$myeloid cells. Conversely, the CM from colonic macrophages in the GA (10 and $20 \mathrm{mg} / \mathrm{kg}$ )-treated mice (GA-CM) significantly inhibited the migration of $\mathrm{Gr}-1^{+}$myeloid cells. HFD-CM increased the arginase activity of $\mathrm{Gr}_{-} 1^{+}$myeloid cells but decreased $\mathrm{CD}^{+}{ }^{+}$cell proliferation, while GA-CM decreased the arginase activity but increased CD8 ${ }^{+} \mathrm{T}$ cell proliferation (Fig. 8b, c). Additionally, Gzm B, IFN- $\gamma$ and IL-2 production was decreased by the HFD-CM but increased by the GA-CM (Fig. 8d). S100A8 and S100A9 expression in the lungs were induced by the HFD-CM but inhibited by the GA-CM (Fig. 8e). Exogenous addition of CCL2 and TNF-a attenuated the inhibitory effect of the GA-CM on $\mathrm{Gr}-1^{+}$myeloid cell migration (Fig. 8f). The inhibition of S100A8 and S100A9 expression in the lungs by the GA-CM was partially reversed by exogenous addition of TNF-a but not CCL2 (Fig. 8g).
LPS/HMGB1/NF-KB signaling is crucial for prevention of premetastatic niche formation by GA

Next, we measured NF-KB signaling in colonic macrophages, and found that the $1 \mathrm{KBa}$ expression was lower in the colonic macrophages from the HFD-fed mice but higher in that from the GA-treated mice. In contrast, p-p65 expression was higher in the macrophages of colon from the GA-treated but lower in that from the HFD-fed mice (Fig. 9a). Similar results of western blotting were observed in the RAW264.7 or THP-1 macrophages treated with the fecal extracts from the mice of the control diet, HFD and HFD plus GA (10 and $20 \mathrm{mg} / \mathrm{kg}$ ) (Fig. 9b, c). Immunofluorescence showed that 065 nuclear translocation was increased by the fecal extracts from the HFD-fed mice, but attenuated by the fecal extracts from the GA-treated mice (Fig. 9d). The release of HMGB1 induced by LPS facilitated M1-like phenotype through the NF-KB signaling. ${ }^{25}$ We found that HMGB1 translocation was increased by the fecal extracts from the HFD-fed mice, but decreased by the fecal extracts from the GA-treated mice (Fig. 9e, f). Next, we measured whether HFD promoted NF-KB signaling through HMGB1. As shown in Fig. 9g, exogenous addition of LPS in the RAW264.7 or THP-1 macrophages increased the p-p65 expression but decreased the IкBa expression, which were attenuated by 
a
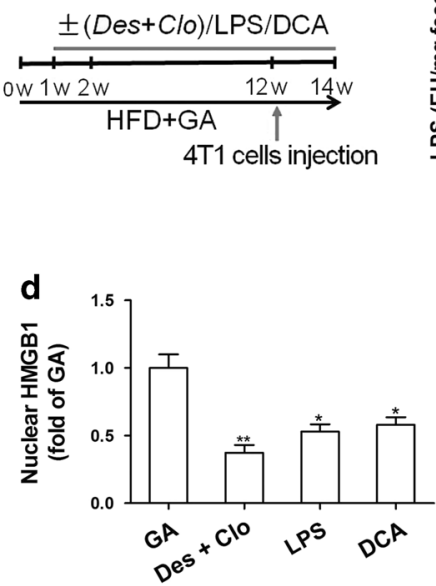

f

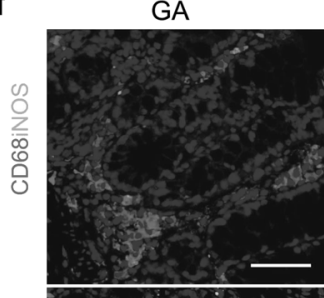

GA
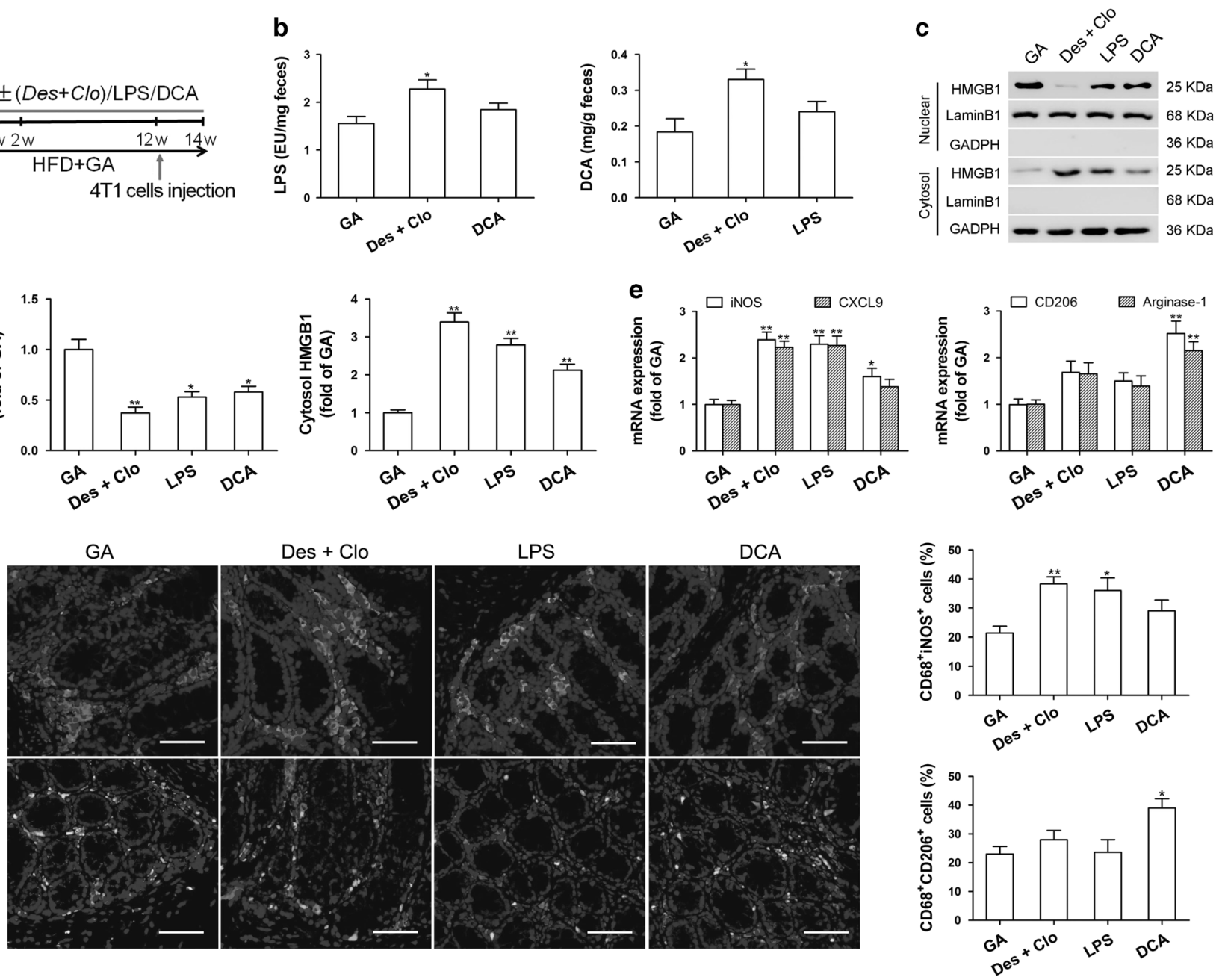

g
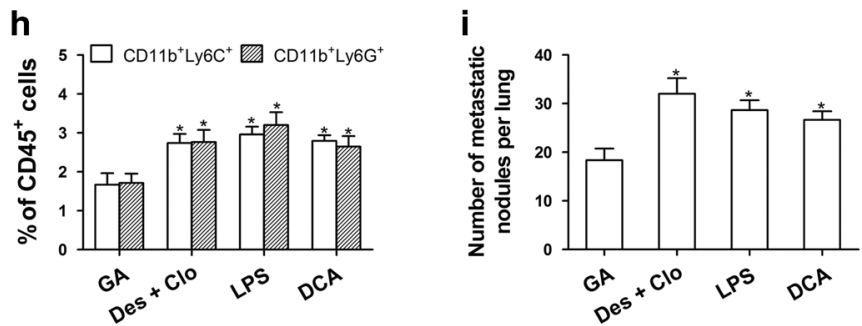

Fig. 5 Macrophage phenotype and their cytokine production are altered by glycyrrhizic acid (GA)-modulated gut microbiota. The Balb/c mice treated with high-fat diet (HFD) plus GA $(20 \mathrm{mg} / \mathrm{kg})$ were administered with bacterial suspension of Desulfovibrio vulgaris (Des) plus Clostridium sordellii (Clo) $\left(1 \times 10^{9} \mathrm{CFU} / \mathrm{mouse}\right.$, i.g., twice a week), or $0.2 \%$ deoxycholic acid (DCA) in drinking water, or lipopolysaccharide (LPS) (300 $\mu \mathrm{g} / \mathrm{kg}$, i. p.) or vehicle for $12 \mathrm{w}$. a Schematic representation of the experimental approach. $\mathbf{b}$ The levels of DCA or LPS in the feces were determined by LC-MS or Limulus Amebocyte Lysate assay, respectively $(n=8)$. c, d HMGB1 in the nuclear and cytoplasmic of colonic macrophages were determined by western blot $(n=8)$. e Relative mRNA levels of M1 markers (iNOS and CXCL9) and M2 markers (CD206 and arginase-1) in the colonic macrophages were measured by qPCR $(n=8)$. $\mathrm{f} I$ mmunofluorescence stained the colon tissues and the quantification the proportion of M1/M2-like macrophages $(n=8)$. g MMP-9, S100A8, and S100A9 in the lungs were determined by western blot $(n=8)$. $\mathbf{h}$ Myeloid-derived suppressor cells in the lungs were analyzed by flow cytometry $(n=5)$. i 4 T1 breast cancer cells $\left(2 \times 10^{5}\right)$ were then injected via vein. At $2 \mathrm{~W}$ after tumor injection, the number of lung metastatic nodules was counted $(n=8)$. The values are presented as the mean $\pm S E M$. ${ }^{*} P<0.05$, $* * P<0.01$ vs. GA

HMGB1 siRNA. Transfection with IkBa siRNA in the RAW264.7 and THP-1 macrophages attenuated the decrease in CCL2 and TNF- $a$ production and $\mathrm{Gr}_{-} 1^{+}$myeloid cell migration mediated by the fecal extracts from the GA-treated mice (Fig. 9h, i).

\section{DISCUSSION}

In the present study, we have provided direct evidence that modulation of gut microbiota by GA suppresses HFD-enhanced pre-metastatic niche formation, and thereby prevents metastasis of $4 \mathrm{~T} 1$ breast cancer and B16F10 melanoma. To our knowledge, this is the first study that directly demonstrated the key role of gut microbiota in pre-metastatic niche formation. In addition, we have demonstrated for the first time that modulation of gut microbiota by GA reprograms colonic macrophages via LPS/HMGB1/NF-KB signaling. Thus, targeting gut microbiota to modulate colonic macrophage response could be a novel strategy for the prevention of pre-metastatic niche formation and metastasis. 

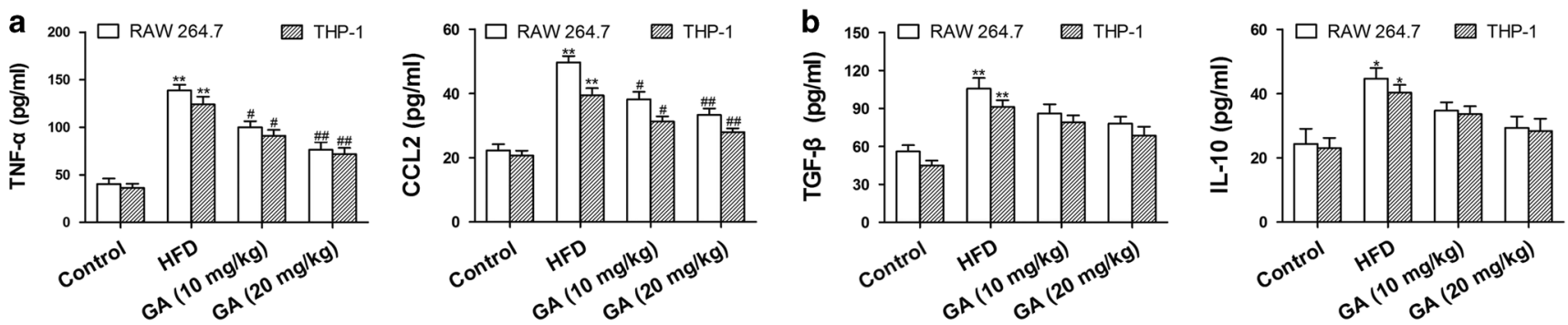

C
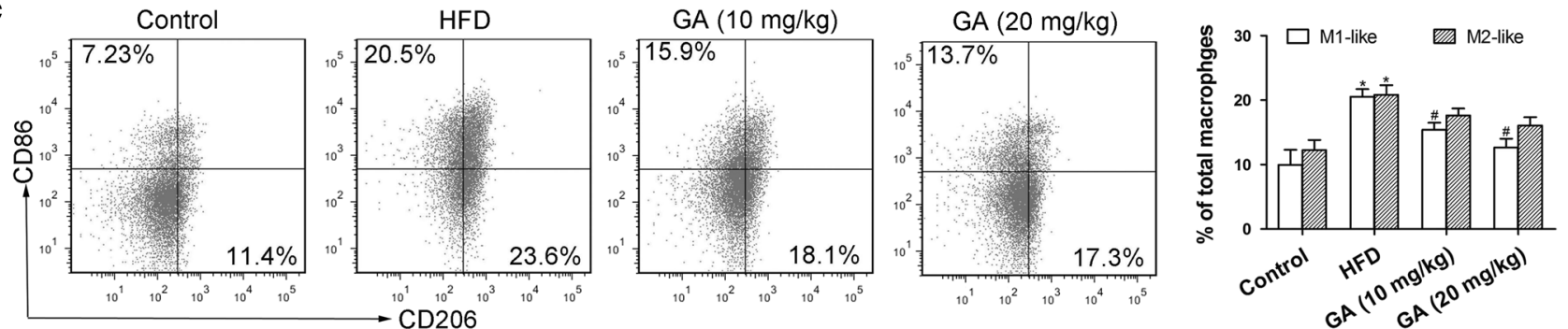

Fig. 6 Macrophage phenotype and their cytokine production are altered by glycyrrhizic acid (GA)-modulated gut microbiota. The RAW264.7 and THP-1 macrophages were treated by the fecal extracts from the control diet, high-fat diet (HFD) and HFD plus GA (10, 20 mg/kg)-treated mice for 12 h. a, b M1 cytokines (TNF- $\alpha$ and CCL2) and M2 cytokines (TGF- $\beta$ and IL-10) in the culture supernatants were determined by ELISA. c Flow cytometry analysis for the proportion of M1- and M2-like macrophages. The values are presented as the mean $\pm S E M$. ${ }^{*} P<0.05$, ${ }^{* *} P<$ 0.01 vs. GA, ${ }^{\#} P<0.05,{ }^{\# \#} P<0.01$ vs. control, ${ }^{\wedge} P<0.05,{ }^{\wedge}{ }^{\wedge} P<0.01$ vs. HFD
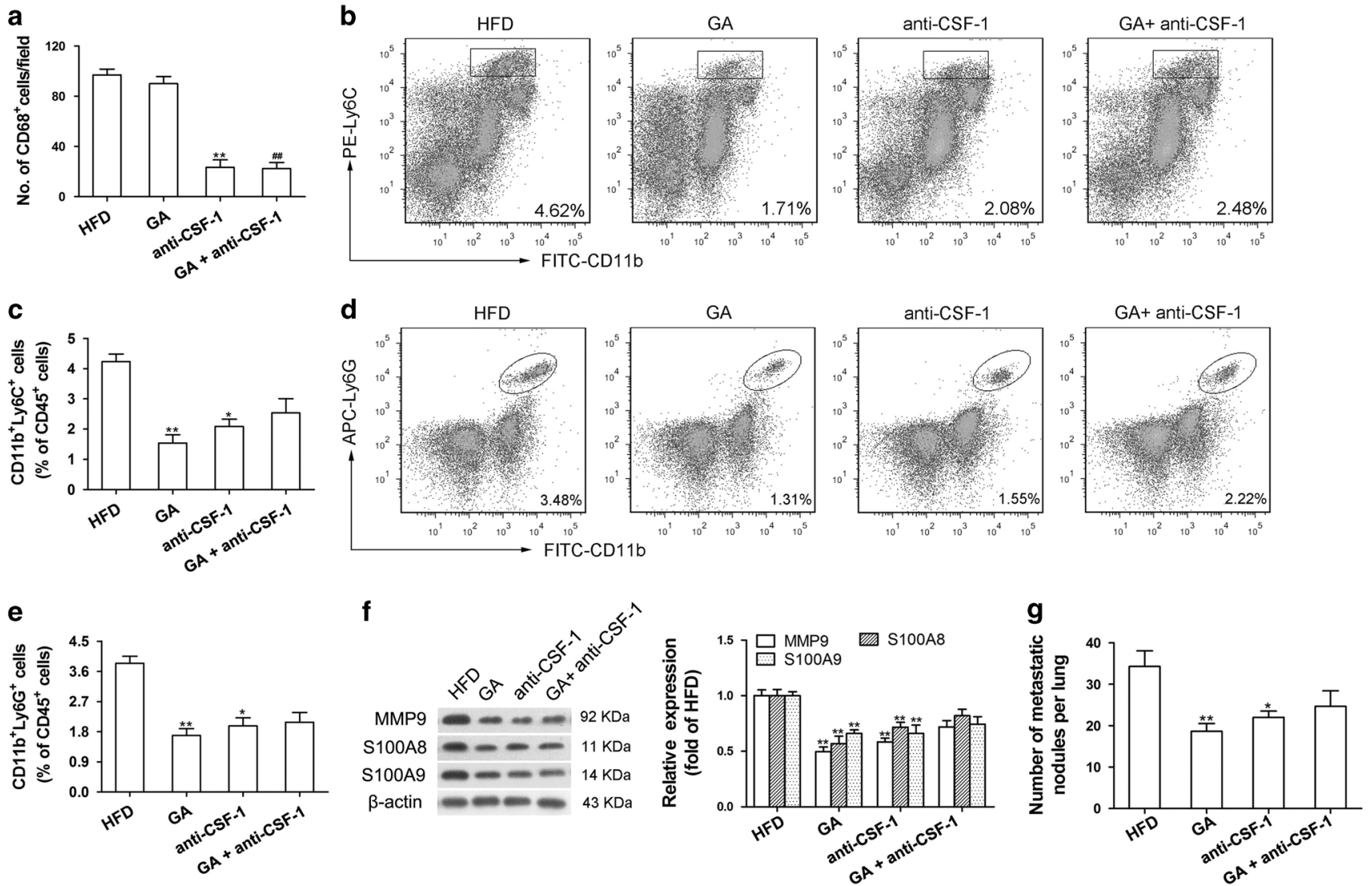

Fig. 7 Glycyrrhizic acid (GA) prevents high-fat diet (HFD)-enhanced lung pre-metastatic niche formation and metastasis through modulation of colonic macrophage phenotype. The Balb/c mice treated with the HFD plus GA $(20 \mathrm{mg} / \mathrm{kg})$ or vehicle were administrated intraperitoneally with anti-CSF-1 antibody $(50 \mathrm{mg} / \mathrm{kg}) 24 \mathrm{~h}$ before the HFD or GA intervention, followed by repeated injections of $25 \mathrm{mg} / \mathrm{kg}$ every 5 days for 12 w. a Immunostaining analysis of the number of CD68 ${ }^{+}$cells $(n=8)$. b-e Flow cytometry analysis for the CD $11 \mathrm{~b}^{+}$Ly $6 \mathrm{G}^{+}$and $C D 11 \mathrm{~b}^{+} \mathrm{Ly} 6 \mathrm{C}^{+}$cells in the lungs $(n=5)$. $\mathbf{f}$ MMP-9, S100A8, and S100A9 in the lungs were determined by western blot $(n=8)$. $\mathbf{g} 411$ breast cancer cells $\left(2 \times 10^{5}\right)$ were then injected via vein. At $2 \mathrm{w}$ after tumor injection, the number of lung metastatic nodules was counted ( $n=8$ ). The values are presented as the mean \pm SEM. ${ }^{*} P<0.05,{ }^{* *} P<0.01$ vs. HFD, ${ }^{\# \#} P<0.01$ vs. GA 

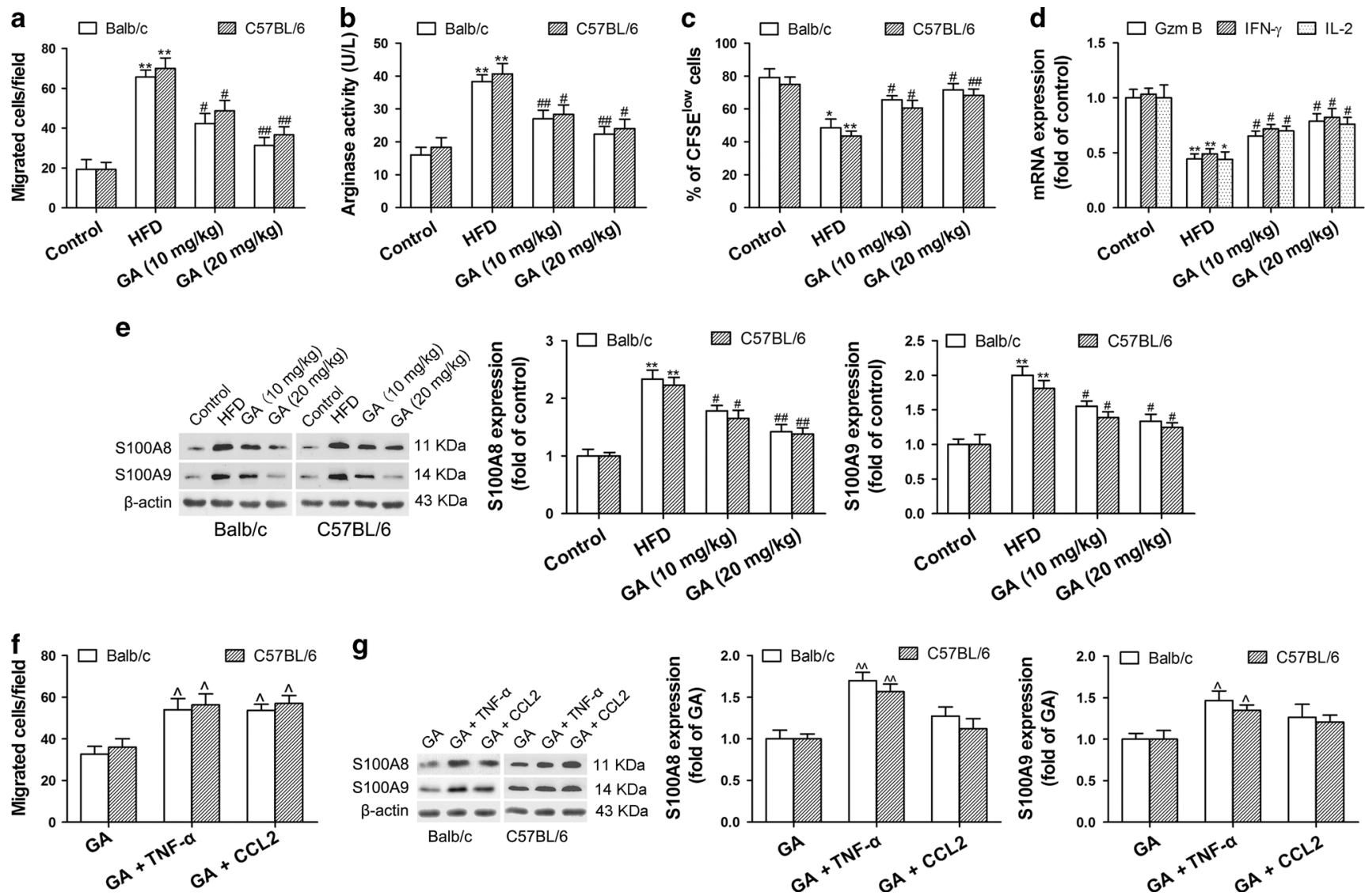

Fig. 8 Glycyrrhizic acid (GA) inhibits pre-metastatic niche formation through colonic macrophage-derived factors. a The conditioned media (CM) from the colonic macrophages in the high-fat diet (HFD) or HFD plus GA-treated mice was added to the lower chamber. Gr-1 ${ }^{+}$myeloid cells were added to the upper chamber, and then incubated $12 \mathrm{~h}$. The cells migrated to the lower chambers were counted. $\mathbf{b}, \mathbf{c}$ The arginase activity and $\mathrm{CD}^{+}{ }^{+}$cell proliferation were determined. $\mathbf{d}$ Relative mRNA levels of Gzm B, IFN- $\gamma$, and IL-2 in the CD8 ${ }^{+} \mathrm{T}_{\text {cell }}$ were measured by qPCR. e The lung tissues from the Balb/c and C57BL/6 mice were stimulated with above CM for $24 \mathrm{~h}$. S100A8 and S100A9 were measured by western blot. $f$ The CM from the colonic macrophages in the HFD plus GA-treated mice was added to the lower chamber with or without exogenous addition of TNF- $\alpha(20 \mathrm{ng} / \mathrm{ml})$ or CCL2 $(10 \mathrm{ng} / \mathrm{ml})$. Gr-1 ${ }^{+}$myeloid cells were added to the upper chamber, and then incubated $12 \mathrm{~h}$. The cells migrated to the lower chambers were counted. $\mathbf{g}$ The lung tissues were stimulated with above CM for $24 \mathrm{~h}$. S100A8 and S100A9 were measured by western blot. Each value represents the mean \pm SEM of three independent triplicate experiments. ${ }^{*} P<0.05$, ${ }^{* *} P<0.01$ vs. control, ${ }^{\#} P<0.05, \# \#<0.01$ vs. HFD, ${ }^{\wedge} P<0.05,{ }^{\wedge} P<<0.01$ vs. GA

HFD contributes to pre-metastatic niche formation and metastasis. ${ }^{19}$ Elimination of pre-metastatic niches prevents tumor metastasis. ${ }^{4}$ The saponin exerts significant anti-metastasis activities in multiple tumors including melanoma. ${ }^{37} \mathrm{GA}$, a triterpenoid saponin glycoside from the licorice roots, inhibits B16 melanoma pulmonary metastasis. ${ }^{30}$ Here we demonstrated that GA (10 and $20 \mathrm{mg} / \mathrm{kg}$ ) prevented HFD-enhanced lung metastasis through inhibiting pre-metastatic niche formation (evidenced by a decrease in the recruitment of MDSCs and the expression of pro-metastatic proteins). Previous study showed that administration of GA $(200 \mathrm{mg} / \mathrm{kg} / \mathrm{d})$ to mice for $96 \mathrm{w}$ does not provide any evidence of chronic toxicity or tumorigenicity, ${ }^{38}$ suggesting that GA prevents HFD-enhanced pre-metastatic niche formation and metastasis with little side effects. Given that tumor-derived cytokines foster tumor growth and metastasis, ${ }^{39}$ further experiments should be performed to elucidate whether GA inhibits premetastatic niche formation and metastasis by targeting tumor microenvironment.

DCA, a kind of secondary bile acids, promotes hepatocellular carcinoma development. ${ }^{17}$ LPS, the major component of gramnegative bacteria cell wall, promotes liver metastasis from colorectal cancer. ${ }^{16}$ Saponins regulate gut microbiota composition and alleviate intestinal inflammatory environment. ${ }^{28}$ Here we demonstrated that HFD increased the abundance of Desulfovibrio genus and clostridiales order, and elevated the levels of LPS and DCA in the feces and serums. Conversely, GA modulated gut microbiota and decreased the levels of LPS and DCA in the feces, and thereby prevented pre-metastatic niche formation and metastasis. Very recently, a study showed that the gut microbiota promotes liver metastasis through translocation of bacterial to the metastatic site. ${ }^{14}$ Further studies could be performed to confirm whether GA inhibits HFD-enhanced pre-metastatic niche formation and metastasis through microbiota translocation.

There are growing evidences suggesting that M1-like macrophages enhance metastatic potential of colon, hepatocellular and ovarian cancer cells. ${ }^{21-23}$ The colonic M1 macrophage is regulated by gut microbiota and its metabolites, ${ }^{40}$ indicating their possible roles in metastasis. Here we demonstrated that HFD activated macrophages in the colons into M1/M2 mixture states with the predominated M1 characteristics. Conversely, GA primarily decreased M1-like macrophages in the colons and then inhibited HFD-enhanced lung pre-metastatic niche formation and metastasis. A previous study showed that anti-inflammatory (M2), but not pro-inflammatory (M1) macrophages promote tumor metastasis. ${ }^{41}$ This discrepancy could be due to differences of pathological conditions (HFD-induce inflammation versus tumorassociated inflammation) and the different niches (colon versus tumor). A variety of tumor-derived factors including CCL2 and 

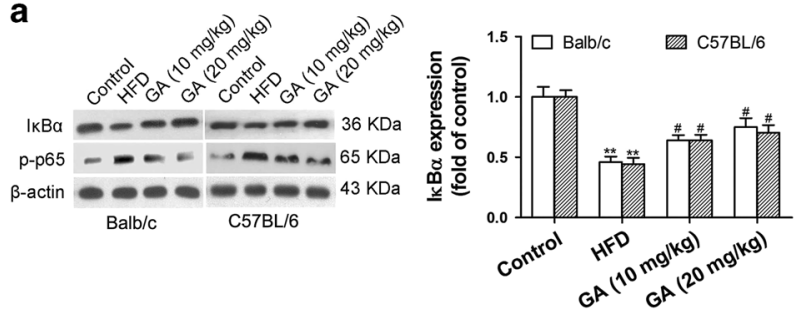

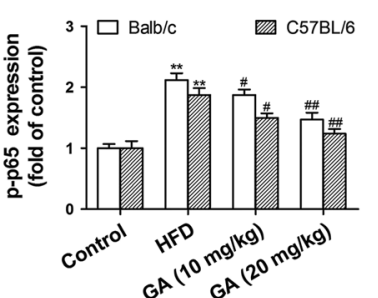

b

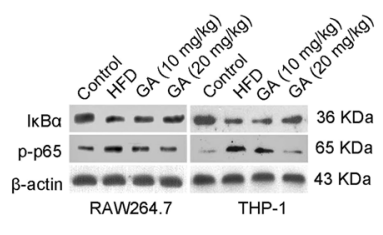

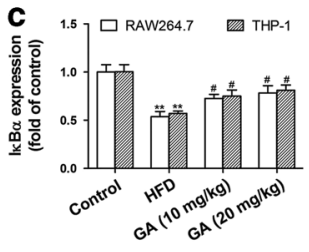

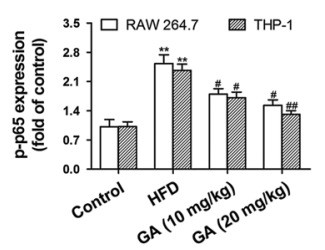

f
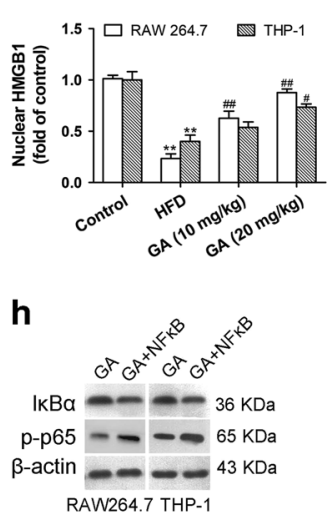

d

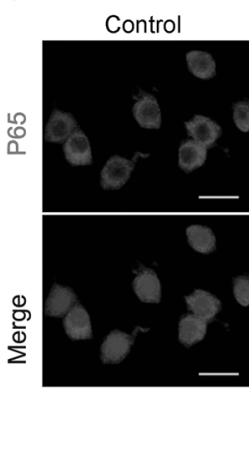

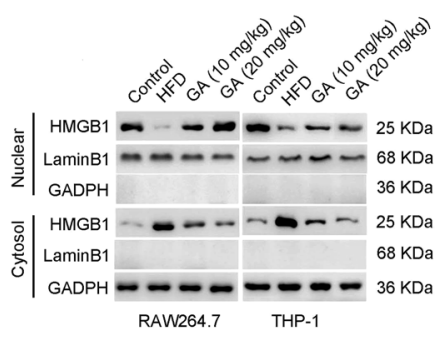
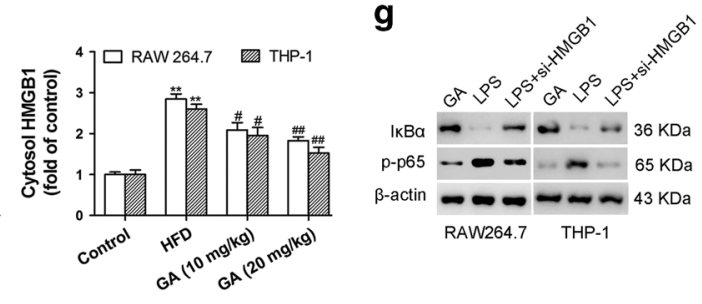
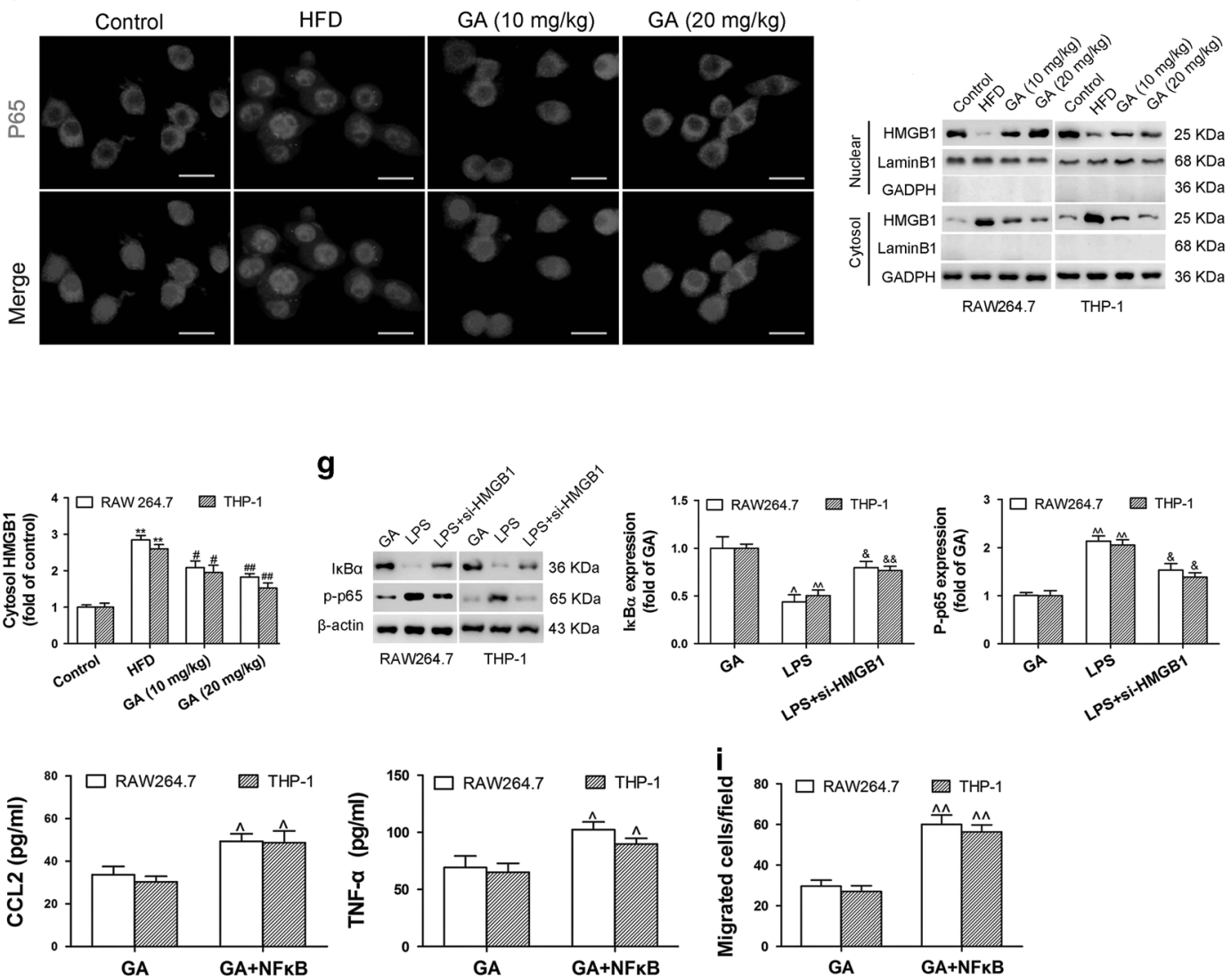

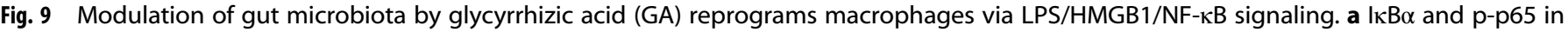
colonic macrophages from the mice of the control diet, high-fat diet (HFD) and HFD plus GA (10 and $20 \mathrm{mg} / \mathrm{kg}$ ) were determined by western blot $(n=8)$. b, c The RAW264.7 and THP-1 macrophages were treated by the fecal extracts from the control, HFD, HFD plus GA (10 and 20 mg/ $\mathrm{kg}$ )-treated mice for $12 \mathrm{~h}$. I $\mathrm{KB} \alpha$ and $\mathrm{p}$-p65 were determined by western blot. $\mathbf{d}$ The localization of p65 was examined by immunofluorescence stain. Scale bar, $20 \mu \mathrm{m}$. e, f Hhigh-mobility group box 1 (HMGB1) in the nuclear and cytoplasmic of the RAW264.7 and THP-1 macrophages were determined by western blot. $\mathbf{g}$ The RAW264.7 and THP-1 macrophages treated with the fecal extracts from the GA-treated mice were administrated with LPS or transfected with HMGB1 lentivirus. IкB $\alpha$ and p-p65 in the RAW264.7 and THP-1 macrophages were determined by western blot. $\mathbf{h}$ The RAW264.7 and THP-1 macrophages transfected with IкB $\alpha$ lentivirus were treated with the fecal extracts from the GAtreated mice, TNF- $\alpha$ and CCL2 in the culture supernatants were determined by ELISA. $\mathbf{i}$ The conditioned media from above groups was added to the lower chamber, $\mathrm{Gr}_{-1}^{+}$myeloid cells were added to the upper chamber, and then incubated $12 \mathrm{~h}$. The cells migrated to the lower chambers were counted. Each value represents the mean \pm SEM of three independent triplicate experiments. ${ }^{*} P<0.05,{ }^{* *} P<0.01$ vs. control, ${ }^{\#} P<0.05,{ }^{\# \#} P<0.01$ vs. HFD, ${ }^{\wedge} P<0.05,{ }^{\wedge \wedge} P<0.01$ vs. GA, ${ }^{\&} P<0.05,{ }^{\& \&} P<0.01$ vs. LPS

TNF-a have been shown to drive the pre-metastatic niche formation. ${ }^{2}$ However, almost nothing has been done on the possible role of colonic macrophages derived cytokines in initiating the process. We demonstrated that the decreased M1 cytokines (CCL2 and TNF-a) from the macrophages in the colons by modulating gut microbiota inhibited pre-metastatic niche formation and metastasis. Previous studies showed that iNOS is commonly used as a marker of recruited macrophages, ${ }^{42}$ and $\mathrm{CCL} 2$ is a key molecule for recruitment of pro-inflammatory macrophages in colon. ${ }^{18}$ Therefore, further studies should be performed to investigate whether newly recruited macrophages not resident macrophages are the major composition of pro- inflammatory M1-like macrophages in the colon from the HFD-fed mice.

The crucial roles of NF-KB in inflammatory process and tumor metastasis have been well documented. ${ }^{43}$ The inhibition of NF-KB signaling exerts anti-metastasis activity. ${ }^{43}$ We demonstrated that HFD activated NF-KB in M1-like macrophages through gut microbiota-derived LPS. Conversely, GA decreased p-p65 level and p65 nuclear translocation. Importantly, NF-KB overexpression by silence of IKBa partly reversed the effects of GA on production of CCL2 and TNF-a and migration of $\mathrm{Gr}^{-} 1^{+}$myeloid cells. Additionally, HMGB1 contributes to melanoma growth and lung metastasis formation. ${ }^{24} \mathrm{GA}$ exerts the anti-flammatory properties 


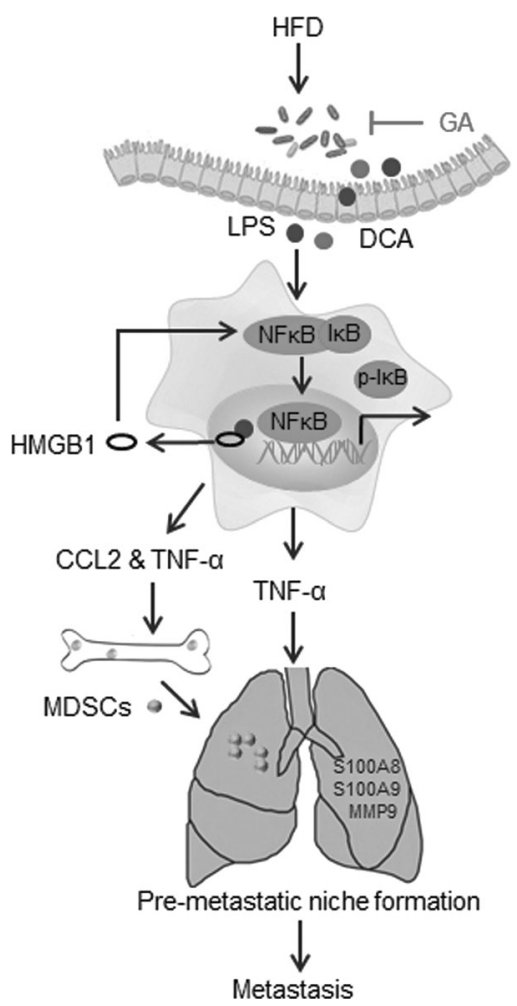

Fig. 10 A proposed mechanism to explain the role of GA-regulated gut microbiota in HFD-enhanced pre-metastatic niche formation and metastasis. HFD high-fat diet, GA glycyrrhizic acid, LPS lipopolysaccharide, HMGB1 high-mobility group box 1, DCA deoxycholic acid, TNF- $\alpha$ tumor necrosis factor- $\alpha$, CCL2 CC chemokine ligand 2, MDSCs myeloid-derived suppressor cells

partly through directly binding to HMGB1. ${ }^{33}$ We demonstrated that GA downregulated microbiota-derived LPS, and thereby inhibited HMGB1 release and greatly inactivated NF-KB. These data suggest that the GA prevents lung pre-metastatic niche formation through gut microbiota-drived LPS/HMGB1/NF-KB signaling in colonic macrophages.

In conclusion, improvement of HFD-mediated gut microbiota dysbiosis by GA prevents pre-metastatic niche formation and metastasis through reprogramming $\mathrm{M} 1$-like macrophages in the colons via LPS/HMGB1/NF-KB signaling (Fig. 10). Importantly, our study identifies previously unknown role of HFD-mediated gut microbiota dysbiosis in pre-metastatic niche formation. Our findings may serve as a potential therapeutic strategy for prevention of pre-metastatic niche formation and metastasis.

\section{MATERIALS AND METHODS}

Chemicals and reagents

GA (G111375) with high purity ( $\geq 98 \%$ ) was purchased from Aladdin. DCA (D2510) with purity $>98 \%$ and LPS (L2880) were purchased from Sigma-Aldrich. Antibodies against CD206 (ab64693), CD68 (ab955), S100A8 (ab180735), S100A9 (ab92507), IKBa (ab7217), HMGB1 (ab79823), GAPDH (ab181602), Lamin B1 (ab133741), Gzm B (ab134933), IL-2 (ab92381), IFN- $\gamma$ (ab9657), pp65 (ab28856), p65 (ab16502), and $\beta$-actin (ab8226) were purchased from Abcam. Antibodies against MMP-9 (sc-13520) and iNOS (sc-651) were purchased from Santa Cruz Biotechnology. Mouse TNF-a (410-MT-010) and CCL2 (479-JE-010) were purchased from R\&D Systems.

Animal models and treatment regimes. Specific pathogen-free Balb/c mice (female, 3 to 4 weeks old) and C57BL/6 mice (male, 3 to 4 weeks old) were purchased from Center for Disease Control and Prevention (Hubei, China). All animal experiments were performed in accordance with the policies of the animal ethics committee of the Animal Research Committee of Wuhan University, and maintained in accordance with the guidelines by the Association for Assessment and Accreditation of Laboratory Animal Care International. All animals were housed at room temperature $\left(18-22^{\circ} \mathrm{C}\right)$ with free access to drinking water under a 12-h light/12-h dark cycle. The mice were fed purified diets (Research Diets Inc.): HFD (D12451) containing $45 \mathrm{kcal} \%$ as fat or the control diet (D12450B) with $10 \mathrm{kcal} \%$ as fat and were caged individually to avoid cage effects. The two diets contained identical quantities of protein, cellulose, soybean oil, vitamins, and minerals per kilocalorie, and only lard and carbohydrates varied between the two diets. The diets were stored at $-20^{\circ} \mathrm{C}$ and fresh diet was provided daily to allow the mice free access to feed. The body weight and food intake was monitored throughout the study.

For pre-metastatic niche formation experiment, Balb/c mice and C57BL/6 mice were fed HFD and treated with GA (10 and $20 \mathrm{mg} / \mathrm{kg}$, i.g., $q d \times 5$ days per $\mathrm{w}$ ) or vehicle for $12 \mathrm{w}$, respectively. The doses of GA used in the present study were based on the published studies $^{44}$ and our preliminary experiments. The mice in the control group were fed the control diet. The lungs, colon, and feces were harvested and analyzed. For spontaneous metastasis experiment, after the HFD or control diet treatment for $12 \mathrm{w}, 4 \mathrm{~T} 1$-luciferase cells $\left(1 \times 10^{6}\right)$ or B16F10-luciferase cells $\left(5 \times 10^{5}\right)$ were injected to the right mammary fat pad of Balb/c mice and the armpit of C57BL/6 mice, respectively. The mice were killed on day 28 after tumor inoculation, and the lungs were collected and analyzed. Lung metastasis was measured by hematoxylin/eosin staining. Pulmonary metastatic nodules were counted in three sections. Lung metastasis was also detected by ex vivo luciferase-based noninvasive bioluminescence imaging system (In-Vivo Xtreme II, Bruker). The lung images were analyzed by quantification of total photon flux of each lung using Molecular Imaging Software.

For antibiotic treatment, the Balb/c mice were received HFD plus GA $(20 \mathrm{mg} / \mathrm{kg})$ or vehicle for $12 \mathrm{w}$, and then treated with a mixture of $0.5 \mathrm{~g} / \mathrm{l}$ vancomycin, $0.5 \mathrm{~g} / \mathrm{l}$ neomycin sulfate and $0.5 \mathrm{~g} / \mathrm{l}$ primaxin in the drinking water for $3 \mathrm{w}$, which was refreshed every $2 \mathrm{~d} .^{45}$ After antibiotic treatment, the mice were killed, and the lungs and feces were harvested and analyzed. Alternatively, 4T1-luciferase breast cancer cells $\left(2 \times 10^{5}\right)$ were injected via vein, lung metastasis was measured on day 14.

Bacteria administration and metabolite supplementation were performed as described. ${ }^{46,47}$ Briefly, a cocktail of lyophilized Desulfovibrio vulgaris (ATCC 29579) plus Clostridium sordellii (ATCC 9714) (1:1) was resuspended in phosphate-buffered saline (PBS) at $5 \times 10^{9} \mathrm{CFU} / \mathrm{ml}$. Each mouse was given $200 \mu$ l of cocktail $\left(1 \times 10^{9}\right.$ CFU/mouse) twice a week by oral gavage. The Balb/c mice fed HFD plus GA $(20 \mathrm{mg} / \mathrm{kg})$ were gavaged with bacterial suspension, or supplemented $0.2 \%$ DCA in drinking water, or injected intraperitoneally LPS $(300 \mu \mathrm{g} / \mathrm{kg})$ or vehicle at $1 \mathrm{w}$. One of the batches was killed after $12 \mathrm{w}$, the feces, colons and lungs were collected and analyzed. The remaining mice were injected with 4T1-luciferase breast cancer cells $\left(2 \times 10^{5}\right)$ via vein, and lung metastasis was measured on day 14.

Macrophages depletion experiment was established as previously described. ${ }^{48}$ Briefly, the Balb/c mice were administrated intraperitoneally with $50 \mathrm{mg} / \mathrm{kg}$ anti-CSF-1 antibody (BioXCell, BE0204) $24 \mathrm{~h}$ before the HFD, followed by repeated injections of $25 \mathrm{mg} / \mathrm{kg}$ every $5 \mathrm{~d}$. After HFD with or without GA $(20 \mathrm{mg} / \mathrm{kg})$ for $12 \mathrm{w}$, the colons and lungs were collected and analyzed. The remaining mice were injected with 4T1-luciferase breast cancer cells $\left(2 \times 10^{5}\right)$ via vein, and lung metastasis was measured on day 14.

Fecal microbiota analysis by $16 \mathrm{~s}$ rRNA sequencing. Fecal microbiota was analyzed by $16 \mathrm{~s}$ rRNA sequencing as described 
previously. ${ }^{49}$ Briefly, fecal samples from the mice treated with the control diet, HFD and HFD plus GA $(20 \mathrm{mg} / \mathrm{kg})$ were freshly collected and immediately frozen in liquidnitrogen. Genomic DNA was isolated from $200 \mathrm{mg}$ frozen fecal samples using a QIAamp DNA Stool Mini Kit (51504, QIAGEN) according to the manufacturer's instructions. Genomic DNA was then amplified in $50 \mu \mathrm{l}$ triplicate reactions with bacterial 16s rRNA gene (V3-V5 region)specific primers: $338 \mathrm{~F}$ (5'-ACTCCTACGGGAGGCAGCAG-3') and 806R (5'-GGACTACHVGGGTWTCTAAT-3'). PCR products were purified and the concentrations were adjusted for sequencing on an Illumina Miseq PE300 system (OEbiotech Co., Ltd.). Pairedend sequences were merged to give an optimal alignment (overlap length $\geq 10 \mathrm{bp}$, mismatch proportion $\leq 20 \%$ ). As an added quality control measure, the software package MacQIIME (version 1.9.1) pipeline was used to filter out and discard poor-quality reads using the default settings. Sequences were further clustered into OTUs (Operational Taxonomic Units or phylotypes) at $97 \%$ of identity using QIIME and cdhit. OTUs were assigned to closest taxonomic neighbors and relative bacterial species using Seqmatch and Blastall. Relative abundance of each OTUs and other taxonomic levels (from phylum to genus) was calculated for each sample to account for different levels of sampling across multiple individuals. Principle Coordinate Analysis ( $\mathrm{PCoA}$ ) projections were visualized using Emperor 0.9.4. To find out the highly-dimensional gut microbes and characterize the differences between two or more biological conditions, we performed the linear discriminant analysis (LDA) effect size (LEfSe) analysis.

Statistical analysis. The values were expressed as mean \pm SEM, and statistical analyses were performed using a one-way ANOVA followed by the Student-Newman-Keuls test. The data of microbiome abundance were analyzed by by nonparametric Mann-Whitney $U$ test correcting for multiple comparisons. $P$ values of 0.05 or less were considered significant.

\section{ACKNOWLEDGEMENTS}

This work was partly supported by the National Natural Science Foundation of China [Grant nos. 81872443, 81272464, and 81173089 (to Jing Yang)].

\section{AUTHOR CONTRIBUTIONS}

M.Q., K.H. and J.Y. conceived and designed the study and wrote the manuscript; M.Q., K.H. and Y.L. performed most of the experiments and analyzed the data; Y.Y., H.T., X.L., C.W. and H.C. conducted animal breeding and sample collection. Y.X. and J.Z. contributed to the statistical analyses. All the authors reviewed the manuscript.

\section{ADDITIONAL INFORMATION}

The online version of this article (https://doi.org/10.1038/s41385-019-0144-6) contains supplementary material, which is available to authorized users.

Competing interests: The authors declare no competing interests.

Publisher's note: Springer Nature remains neutral with regard to jurisdictional claims in published maps and institutional affiliations.

\section{REFERENCES}

1. Gilkes, D. M., Semenza, G. L. \& Wirtz, D. Hypoxia and the extracellular matrix: drivers of tumour metastasis. Nat. Rev. Cancer 14, 430-439 (2014).

2. Peinado, H. et al. Pre-metastatic niches: organ-specific homes for metastases. Nat. Rev. Cancer 17, 302-317 (2017).

3. Liu, Y. \& Cao, X. Characteristics and significance of the pre-metastatic niche. Cancer Cell. 30, 668-681 (2016).

4. Kowanetz, M. et al. Granulocyte-colony stimulating factor promotes lung metastasis through mobilization of $\mathrm{Ly} 6 \mathrm{G}^{+} \mathrm{Ly} 6 \mathrm{C}^{+}$granulocytes. Proc. Natl Acad. Sci. USA 107, 21248-21255 (2010).

5. Parekh, N., Chandran, U. \& Bandera, E. V. Obesity in cancer survival. Annu. Rev. Nutr. 32, 311-342 (2012)
6. Zimmet, P., Alberti, K. G. \& Shaw, J. Global and societal implications of the diabetes epidemic. Nature 414, 782-787 (2001).

7. Magaki, M. et al. A high-fat diet increases the incidence of mammary cancer incHa-ras proto-oncogene transgenic rats. J. Toxicol. Pathol. 30, 145-152 (2017).

8. Pandey, V., Vijayakumar, M. V., Ajay, A. K., Malvi, P. \& Bhat, M. K. Diet-induced obesity increases melanoma progression: involvement of Cav-1 and FASN. Int. J. Cancer 130, 497-508 (2012).

9. Jung, J. I. et al. High-fat diet-induced obesity increases lymphangiogenesis and lymph node metastasis in the B16F10 melanoma allograft model: roles of adipocytes and M2-macrophages. Int. J. Cancer 136, 258-270 (2015).

10. Kim, E. J. et al. Dietary fat increases solid tumor growth and metastasis of $4 \mathrm{~T} 1$ murine mammary carcinoma cells and mortality in obesity-resistant BALB/c mice. Breast Cancer Res. 13, R78 (2011).

11. Quail, D. F. et al. Obesity alters the lung myeloid cell landscape to enhance breast cancer metastasis through IL5 and GM-CSF. Nat. Cell Biol. 19, 974-987 (2017).

12. Schulz, M. D. et al. High-fat-diet-mediated dysbiosis promotes intestinal carcinogenesis independently of obesity. Nature 514, 508-512 (2014).

13. Wong, S. H. et al. Gavage of fecal samples from patients with colorectal cancer promotes intestinal carcinogenesis in germ-free and conventional mice. Gastroenterology 153, 1621-1633.e6 (2017).

14. Bullman, S. et al. Analysis of Fusobacterium persistence and antibiotic response in colorectal cancer. Science 358, 1443-1448 (2017).

15. Li, J. et al. Probiotics modulated gut microbiota suppresses hepatocellular carcinoma growth in mice. Proc. Natl Acad. Sci. USA 113, E1306-E1315 (2016).

16. El, R. T. et al. Lung inflammation promotes metastasis through neutrophil protease-mediated degradation of Tsp-1. Proc. Natl Acad. Sci. USA 112, 16000-16005 (2015).

17. Yoshimoto, S. et al. Obesity-induced gut microbial metabolite promotes liver cancer through senescence secretome. Nature 499, 97-101 (2013).

18. Kawano, Y. et al. Colonic pro-inflammatory macrophages cause insulin resistance in an intestinal Ccl2/Ccr2-dependent manner. Cell Metab. 24, 295-310 (2016).

19. Nagahashi, M. et al. Targeting the SphK1/S1P/S1PR1 axis that links obesity, chronic inflammation, and breast cancer metastasis. Cancer Res. 78, 1713-1725 (2018).

20. $\mathrm{Hu}, \mathrm{X}$. et al. Microglial and macrophage polarization-new prospects for brain repair. Nat. Rev. Neurol. 11, 56-64 (2015).

21. Jedinak, A., Dudhgaonkar, S. \& Sliva, D. Activated macrophages induce metastatic behavior of colon cancer cells. Immunobiology 215, 242-249 (2010).

22. Wang, H. et al. CD68(+)HLA-DR(+) M1-like macrophages promote motility of HCC cells via NF-KB/FAK pathway. Cancer Lett. 345, 91-99 (2014).

23. Cho, U., Kim, B., Kim, S., Han, Y. \& Song, Y. S. Pro-inflammatory M1 macrophage enhances metastatic potential of ovarian cancer cells through NF-KB activation. Mol. Carcinog. 57, 235-242 (2018).

24. Bianchi, M. E. et al. High-mobility group box 1 protein orchestrates responses to tissue damage via inflammation, innate and adaptive immunity, and tissue repair. Immunol. Rev. 280, 74-82 (2017).

25. Huber, R. et al. Tumour hypoxia promotes melanoma growth and metastasis via High Mobility Group Box-1 and M2-like macrophages. Sci. Rep. 6, 29914 (2016).

26. Friedman, J. E. et al. Pyrroloquinoline quinone prevents developmental programming of microbial dysbiosis and macrophage polarization to attenuate liver fibrosis in offspring of obese mice. Hepatol. Commun. 2, 313-328 (2018).

27. DHJ, C. et al. Molecular targets and anti-cancer potential of escin. Cancer Lett. 422, 1-8 (2018)

28. Dong, W. W. et al. Comparative analysis of the rats' gut microbiota composition in animals with different ginsenosides metabolizing activity. J. Agric. Food Chem. 65, 327-337 (2017).

29. Kim, K. J., Choi, J. S., Kim, K. W. \& Jeong, J. W. The anti-angiogenic activities of glycyrrhizic acid in tumor progression. Phytother. Res. 27, 841-846 (2013).

30. Kobayashi, M. et al. Inhibitory effect of glycyrrhizin on experimental pulmonary metastasis in mice inoculated with B16 melanoma. Anticancer Res. 22, 4053-4058 (2002).

31. Wang, C. et al. Protective effects of glycyrrhizic acid from edible botanical glycyrrhiza glabra against non-alcoholic steatohepatitis in mice. Food Funct. 7, 3716-3723 (2016)

32. Abo, E. N. F., El-Mesery, M., El-Karef, A. \& El-Shishtawy, M. M. Glycyrrhizin ameliorates high fat diet-induced obesity in rats by activating NrF2 pathway. Life. Sci. 193, 159-170 (2018).

33. Mollica, L. et al. Glycyrrhizin binds to high-mobility group box 1 protein and inhibits its cytokine activities. Chem. Biol. 14, 431-441 (2007).

34. Ritz, N. L., Lin, D. M., Wilson, M. R., Barton, L. L. \& Lin, H. C. Sulfate-reducing bacteria slow intestinal transit in a bismuth-reversible fashion in mice. Neurogastroenterol. Motil. 29, e12907 (2017).

35. Figueiredo, M. C. et al. Hybrid cluster proteins and flavodiiron proteins afford protection to Desulfovibrio vulgaris upon macrophage infection. J. Bacteriol. 195, 2684-2690 (2013). 
36. Chen, X. W. et al. CYP4A in tumor-associated macrophages promotes premetastatic niche formation and metastasis. Oncogene 36, 5045-5057 (2017).

37. $\mathrm{Xu}, \mathrm{X}$. H. et al. Saponins from chinese medicines as anticancer agents. Molecules 21, 1326 (2016).

38. Isbrucker, R. A. \& Burdock, G. A. Risk and safety assessment on the consumption of Licorice root (Glycyrrhiza sp.), its extract and powder as a food ingredient, with emphasis on the pharmacology and toxicology of glycyrrhizin. Regul. Toxicol. Pharmacol. 46, 167-192 (2006).

39. McAllister, S. S. \& Weinberg, R. A. The tumour-induced systemic environment as a critical regulator of cancer progression and metastasis. Nat. Cell Biol. 16, 717-727 (2014).

40. Isidro, R. A. \& Appleyard, C. B. Colonic macrophage polarization in homeostasis, inflammation, and cancer. Am. J. Physiol. Gastrointest. Liver Physiol. 311, G59-G73 (2016).

41. Rolny, C. et al. HRG inhibits tumor growth and metastasis by inducing macrophage polarization and vessel normalization through downregulation of PIGF. Cancer Cell. 19, 31-44 (2011).

42. Mowat, A. M. \& Bain, C. C. Mucosal macrophages in intestinal homeostasis and inflammation. J. Innate Immun. 3, 550-564 (2011).
43. Bilandzic, M. et al. Betaglycan blocks metastatic behaviors in human granulosa cell tumors by suppressing NFKB-mediated induction of MMP2. Cancer Lett. 354 107-114 (2014).

44. Wang, D. et al. 18ß-glycyrrhetinic acid, a novel naturally derived agent, suppresses prolactin hyperactivity and reduces antipsychotic-induced hyperprolactinemia in in vitro and in vivo models. Neurochem. Res. 41, 2233-2242 (2016).

45. lida, N. et al. Commensal bacteria control cancer response to therapy by modulating the tumor microenvironment. Science 342, 967-970 (2013).

46. Cao, H. et al. Secondary bile acid-induced dysbiosis promotes intestinal carcinogenesis. Int. J. Cancer 140, 2545-2556 (2017).

47. Sivan, A. et al. Commensal Bifidobacterium promotes antitumor immunity and facilitates anti-PD-L1 efficacy. Science 350, 1084-1089 (2015).

48. Ruffell, B. et al. Macrophage IL-10 blocks $\mathrm{CD} 8^{+} \mathrm{T}$ cell-dependent responses to chemotherapy by suppressing IL-12 expression in intratumoral dendritic cells. Cancer Cell. 26, 623-637 (2014).

49. Chang, C. J. et al. Corrigendum: Ganoderma lucidum reduces obesity in mice by modulating the composition of the gut microbiota. Nat. Commun. 8, 16130 (2017). 